\title{
Topological properties of the binary supremum function
}

\author{
Peter Hertling ${ }^{1}$
}

Received: 27 October 2021 / Accepted: 18 January 2022 / Published online: 23 February 2022

(c) The Author(s) 2022

\begin{abstract}
We consider the binary supremum function sup : $Z \times Z \rightarrow Z$ on a sup semilattice $Z$ and its topological properties with respect to the Scott topology and the product topology. It is well known that this function is continuous with respect to the Scott topology on $Z \times Z$. We show that it is open as well. Isbell has constructed several examples of complete lattices $Z$ such that the binary supremum function on $Z$ is discontinuous with respect to the product topology. Of course, in these cases the Scott topology on $Z \times Z$ is strictly finer than the product topology. This raises the question whether there exists a complete lattice $Z$ such that the Scott topology on $Z \times Z$ is strictly finer than the product topology and such that the binary supremum function is continuous even with respect to the product topology. We construct such a lattice. Finally, by using any of the examples constructed by Isbell, we show the following result: Bounded completeness of a complete lattice $Z$ is in general not inherited by the dcpo $C(X, Z)$ of continuous functions from $X$ to $Z$ where $X$ is a topological space and where on $Z$ the Scott topology is considered. On the other hand, we show that bounded completeness of $Z$ is inherited by $C(X, Z)$ if the topology on $X$ is the Scott topology of a partial order.
\end{abstract}

Keywords Sup semilattice $\cdot$ Supremum function $\cdot$ Scott topology $\cdot$ Product topology $\cdot$ Continuity $\cdot$ Open functions $\cdot$ Bounded completeness

\section{Introduction}

In this article two examples in the theory of partial orders are constructed. The first example concerns the question whether the binary supremum function on a sup semilattice is continuous with respect to the product topology of the Scott topology on each

Communicated by Jimmie D. Lawson.

Peter Hertling

peter.hertling@unibw.de

1 Fakultät für Informatik, Universität der Bundeswehr München, Werner-Heisenberg-Weg 39, 85577 Neubiberg, Germany 
copy of the sup semilattice. The second example is a counterexample concerning the question whether bounded completeness of a complete lattice is inherited by the dcpo of continuous functions from some topological space to the complete lattice.

The Scott topology on a partially ordered set (short: poset) $(X, \leq)$ has turned out to be important in many areas of computer science. Given two posets $\left(X, \leq_{X}\right)$ and $\left(Y, \leq_{Y}\right)$ we have two natural topologies on the product poset $\left(X \times Y, \leq_{X \times Y}\right)$ :

- The product topology of the Scott topology on $\left(X, \leq_{X}\right)$ and of the Scott topology on $\left(Y, \leq_{Y}\right)$,

- the Scott topology on $\left(X \times Y, \leq_{X \times Y}\right)$.

It is well known that the second topology on $X \times Y$ is always at least as fine as the first topology, and that there are examples where the second topology is strictly finer than the first topology. The best known example of this kind seems to consist of the Johnstone space (compare [12]) for $(X, \leq)$ and the dcpo of its Scott open subsets for $(Y, \leq)$; see [6, Exercise II-4.26 and Exercise II.1-36] and [7, Exercise 5.2.16 and Exercise 5.2.15]. Other examples are the complete lattices constructed by Isbell $[10,11]$.

If the supremum of any two elements of a poset $(X, \leq)$ exists (in this case the poset is called a sup semilattice) then the binary supremum function $\sup ^{(2)}: X \times X \rightarrow X$ is well defined. Is it continuous? It is easy to see and well known that it is continuous if on $X \times X$ the finer topology of the two topologies discussed above, the Scott topology, is considered. Isbell $[10,11]$ has constructed several examples of complete lattices such that the binary supremum function is discontinuous with respect to the product topology on $X \times X$. Of course, in these cases the Scott topology on $Z \times Z$ is strictly finer than the product topology. This raises the question (posed by an anonymous referee of [8]) whether perhaps the binary supremum function $\sup ^{(2)}: X \times X \rightarrow X$ is always discontinuous with respect to the product topology on $X \times X$ if this is different from the Scott topology on $X \times X$. We answer this question negatively by providing an example of a complete lattice $X$ such that the Scott topology on $X \times X$ is strictly finer than the product topology but nevertheless the function $\sup ^{(2)}: X \times X \rightarrow X$ is continuous with respect to the product topology on $X \times X$; see Theorem 7.4.

Our second example concerns bounded completeness of the dcpo of continuous functions from a topological space to a bounded complete dcpo. A poset is called bounded complete if each bounded subset has a supremum. The following question arises: If $(Z, \leq)$ is a bounded complete dcpo and $X$ a topological space, is then the depo $C(X, Z)$ of continuous functions from $X$ to $Z$ (where on $Z$ the Scott topology is considered) bounded complete as well? It turns out that the answer to this question is yes if the topology on $X$ is the Scott topology of some partial order on $X$. One may now speculate whether the answer is yes even for arbitrary topological spaces. But it is the second main goal of this article to show that in general the answer to this question is no. In Sect. 8 we shall even construct a topological space $X$ and a complete lattice $Z$ such that the dcpo of continuous functions from $X$ to $Z$ is not bounded complete; see Theorem 8.5. This construction makes essential use of a complete lattice $X$ such that the binary supremum function $\sup ^{(2)}: X \times X \rightarrow X$ is discontinuous with respect to the product topology, as constructed by Isbell $[10,11]$ (Theorem 7.1). Note that in [13, Page 26] the closely related question is discussed whether the pointwise supremum 
of two continuous functions $f, g: X \rightarrow Z$ for a topological space $X$ and a complete lattice $Z$ is again continuous. The authors observe that this is true if the product topology on $Z \times Z$ is identical with the Scott topology on $Z \times Z$. Concerning the general case, they write "But in general, we cannot conclude that $f \vee g$ or $f \wedge g$ are Scott continuous". But they do not give an example where the pointwise supremum $f \vee g$ of $f$ and $g$ is not continuous. In Theorem 8.5, the main result of Sect. 8, such an example is constructed.

After introducing some notation and collecting several elementary notions concerning partial orders in the following two sections, in Sect. 4 we modify the Johnstone space in order to obtain a considerably simpler partial order which is even a complete lattice and has additional properties that will be needed in the proof of Theorem 7.4 in Sect. 7. In Sect. 5 we first remind the reader of some elementary algebraic properties of the binary supremum function $\sup ^{(2)}: X \times X \rightarrow X$ and then show that it is not only continuous with respect to the Scott topology on $X \times X$ but also an open mapping with respect to the Scott topology on $X \times X$. In Sect. 6 we present an observation by Wei and Shouli [14] saying that for certain posets one can characterize the property "sobriety" by a condition on the binary supremum function. In Sect. 7, first we shortly speak about the constructions by Isbell [10,11] of complete lattices $(X, \leq)$ such that the function $\sup ^{(2)}: X \times X \rightarrow X$ is not continuous with respect to the product topology on $X \times X$. Then we construct a complete lattice $(X, \leq)$ such that the function $\sup ^{(2)}: X \times X \rightarrow X$ is continuous with respect to the product topology on $X \times X$ although this topology is different from the Scott topology on $X \times X$. Finally, in Sect. 8, first we show that for any poset $X$ and any bounded complete dcpo $Z$ the dcpo $C(X, Z)$ of continuous functions from $X$ to $Z$ is bounded complete as well. Then we construct a topological space $X$ and a complete lattice $Z$ such that the dcpo of continuous functions from $X$ to $Z$ is not bounded complete and such that there exist two continuous functions $f, g: X \rightarrow Z$ whose pointwise supremum is not continuous.

\section{Some notations}

Let $\mathbb{N}:=\{0,1,2, \ldots\}$ denote the set of natural numbers, that is, of non-negative integers. For $k \in \mathbb{N}$ and a total function $f: \mathbb{N} \rightarrow \mathbb{N}$ let

$$
f \nmid k:\{0,1, \ldots, k-1\} \rightarrow \mathbb{N}
$$

be the restriction of the function $f$ to the set $\{0,1, \ldots, k-1\}$. For sets $X, Y$, a function $f: X \rightarrow Y$ and subsets $S \subseteq X, T \subseteq Y$ we write

$$
\begin{aligned}
f^{-1}[T] & :=\{x \in X: f(x) \in T\}, \\
f[S] & :=\{y \in Y:(\exists s \in S) f(s)=y\} .
\end{aligned}
$$

For sets $X, Y$ the projections $\pi_{1}: X \times Y \rightarrow X$ and $\pi_{2}: X \times Y \rightarrow Y$ are defined as usual by $\pi_{1}(x, y):=x$ and $\pi_{2}(x, y):=y$, for $(x, y) \in X \times Y$. 


\section{Basic notions: partial orders and topology}

In this section, for the convenience of the reader, we collect several elementary notions concerning partial orders and topological spaces, in particular the definition of the Scott topology.

Let $(Z, \leq)$ be a poset. For a subset $S \subseteq Z$ we define $\uparrow S:=\{z \in Z:(\exists y \in S) y \leq$ $z\}$ and $\downarrow S:=\{z \in Z:(\exists y \in S) z \leq y\}$. A subset $S \subseteq Z$ is called upwards closed if $S=\uparrow S$. It is called downwards closed if $S=\downarrow S$. An element $z \in Z$ is called an upper bound of a subset $S \subseteq Z$ if $S \subseteq \downarrow\{z\}$. An element $z \in Z$ is called a supremum or least upper bound of a subset $S \subseteq Z$ if it is an upper bound of $S$ and if for all upper bounds $y$ of $S$ one has $z \leq y$. Obviously, if a subset $S \subseteq Z$ has a supremum, then this supremum is unique. Then we denote it by $\sup _{\leq}(S)$ or simply by $\sup (S)$ if it is clear which relation $\leq$ is meant. An element $z \in Z$ is called a lower bound of a subset $S \subseteq Z$ if $S \subseteq \uparrow\{z\}$. An element $z \in Z$ is called an infimum or greatest lower bound of a subset $S \subseteq Z$ if it is a lower bound of $S$ and if for all lower bounds $y$ of $S$ one has $y \leq z$. Obviously, if a subset $S \subseteq Z$ has an infimum, then this infimum is unique. Then we denote it by $\inf _{\leq}(S)$ or simply by $\inf (S)$. The poset $(Z, \leq)$ is called a sup semilattice if $\sup (\{x, y\})$ exists for all $x, y \in Z$. The poset $(Z, \leq)$ is called a complete lattice if $\sup (S)$ exists for all subsets $S \subseteq Z$. Note that then also $\inf (S)$ exists for all subsets $S \subseteq Z$. Indeed, it is well known-compare, e.g., [6, Proposition O-2.2(i)]— and straightforward to check that if $\sup (S)$ exists for all subsets $S \subseteq Z$ then for any subset $T \subseteq Z$, the supremum of the set of all lower bounds of $T$ is an infimum of $T$. A subset $S \subseteq Z$ is called directed if it is nonempty and for any two elements $x, y \in S$ there exists an upper bound $z \in S$ of the set $\{x, y\}$. The poset $(Z, \leq)$ is called a dcpo if for any directed subset $S \subseteq Z$ there exists a supremum of $S$ in $Z$. It is clear that any complete lattice is a dcpo. In Sect. 6 we will consider posets that are at the same time a sup semilattice and a dcpo. Such posets can be characterized as follows.

Lemma 3.1 For a poset $(Z, \leq)$ the following two conditions are equivalent.

1. It is a sup semilattice and a dcpo.

2. For every nonempty subset $S \subseteq Z$ the supremum $\sup (S)$ exists.

Proof " $2 \Rightarrow 1$ ": Trivial. " $1 \Rightarrow 2$ ": Let us assume that $(Z, \leq)$ is a sup semilattice and a dcpo. Let $S \subseteq Z$ be a nonempty subset of $Z$. We wish to show that $\sup (S)$ exists. The set

$$
D:=\{z \in Z \mid(\exists F \subseteq S)(F \text { is finite and } z=\sup (F))\}
$$

is a directed subset of $Z$. Therefore, $\sup (D)$ exists. Obviously, an element $z \in Z$ is an upper bound of $D$ if, and only if, it is an upper bound of $S$. Hence, $\sup (D)$ is a least upper bound of $S$ as well, that means, $\sup (S)$ exists and is equal to $\sup (D)$.

Example 3.2 Let $(X, \tau)$ be a topological space. Then $(\tau, \subseteq)$ is a complete lattice and, for any subset $\sigma \subseteq \tau, \sup (\sigma)=\bigcup \sigma[6$, Examples O-2.7(3)].

Next, we introduce the Scott topology. 
Lemma 3.3 (See, e.g., [1, Definition 2.3.1 and Proposition 2.3.2] [6, Section II-1] or [7, Prop. 4.2.18]) Let $(Z, \leq)$ be a poset. The set of all subsets $U \subseteq Z$ satisfying the following two conditions:

1. $U$ is upwards closed,

2. for every directed subset $S \subseteq Z$ such that $\sup (S)$ exists and $\sup (S) \in U$ the intersection $S \cap U$ is not empty,

is a $T_{0}$ topology on $Z$.

The topology described in this lemma is called the Scott topology of $(Z, \leq)$. Its elements are called Scott open. Let us denote this topology by $\sigma(Z, \leq)$. For a topological space $X$ and a point $x_{0} \in X$ let $\operatorname{cl}\left(\left\{x_{0}\right\}\right)$ be the closure of the set $\left\{x_{0}\right\}$, that is, the intersection of all closed sets containing $x_{0}$ (thus, $\operatorname{cl}\left(\left\{x_{0}\right\}\right)$ is the smallest closed set containing $x_{0}$ ). The following example of a Scott open set is useful for the proof of the assertion in Lemma 3.3 that the Scott topology is $T_{0}$ and will be useful later as well.

Example 3.4 Let $(Z, \leq)$ be a poset. Then for any $y \in Z$ the set

$$
Z \backslash \downarrow\{y\}=\{z \in Z: z \not \leq y\},
$$

is Scott open. In fact, $\downarrow\{y\}=\operatorname{cl}(\{y\})$.

\section{Products of partial orders}

Let $\left(X, \leq_{X}\right)$ and $\left(Y, \leq_{Y}\right)$ be two posets. On the product $Z:=X \times Y$ we define a binary relation $\leq X \times Y \subseteq Z \times Z$ by

$$
(x, y) \leq_{X \times Y}\left(x^{\prime}, y^{\prime}\right): \Longleftrightarrow\left(x \leq_{X} x^{\prime} \text { and } y \leq_{Y} y^{\prime}\right)
$$

for any $x, x^{\prime} \in X$ and $y, y^{\prime} \in Y$.

Lemma 4.1 1. $\left(X \times Y, \leq_{X \times Y}\right)$ is a poset as well.

2. If $S \subseteq X \times Y$ is a directed subset then the sets $\pi_{1}[S] \subseteq X$ and $\pi_{2}[S] \subseteq Y$ are directed as well.

3. For any subset $S \subseteq X \times Y$ the following two conditions are equivalent:

(a) The suprema $\sup _{\leq_{X}}\left(\pi_{1}[S]\right)$ and $\sup _{\leq_{Y}}\left(\pi_{2}[S]\right)$ exist,

(b) The supremum $\sup _{\leq X \times Y}(S)$ exists.

If one, and the both, of these two conditions are satisfied then $\sup _{\leq_{X \times Y}}(S)=$ $\left(\sup _{\leq X}\left(\pi_{1}[S]\right), \sup _{\leq Y}\left(\pi_{2}[S]\right)\right)$.

4. If $\left(X, \leq_{X}\right)$ and $\left(Y, \leq_{Y}\right)$ are complete lattices then $\left(X \times Y, \leq_{X \times Y}\right)$ is a complete lattice as well.

Proof It is obvious that $\leq_{X \times Y}$ is a partial order. The second and the third assertion are straightforward to check as well. And the fourth assertion follows directly from the first and the third assertion. 
For two posets $\left(X, \leq_{X}\right)$ and $\left(Y, \leq_{Y}\right)$, we have two natural topologies on the product $X \times Y$ : the Scott topology $\sigma\left(X \times Y, \leq_{X \times Y}\right)$ and the product topology $\sigma\left(X, \leq_{X}\right)$ $\times \sigma\left(Y, \leq_{Y}\right)$. What is the relation between these two topologies? It is well-known that the Scott topology is always at least as fine as the product topology.

Lemma 4.2 ( [6, Page 197] or [7, Exercise 4.5.19]) For any two posets $\left(X, \leq_{X}\right)$ and $\left(Y, \leq_{Y}\right)$, every subset of $X \times Y$ that is open in the product topology is Scott open as well.

It is also well known that in general the converse of Lemma 4.2 is not true.

Proposition 4.3 There exist dcpo's $\left(X, \leq_{X}\right)$ and $\left(Y, \leq_{Y}\right)$ such that the Scott topology on $\left(X \times Y, \leq_{X \times Y}\right)$ is strictly finer than the product topology on $X \times Y$ of the Scott topology on $\left(X, \leq_{X}\right)$ and the Scott topology on $\left(Y, \leq_{Y}\right)$.

The standard example seems to be $\left(X, \leq_{X}\right):=$ the so-called Johnstone space, $\left(Y, \leq_{Y}\right):=$ the complete lattice of the Scott open subsets of $X$ with the subset relation; compare [6, Exercise II-4.26 and Exercise II.1-36] and [7, Exercises 5.2.16 and 5.2.15]. Since in the following we are going to define a similar but simpler partial order (that is even a complete lattice) for comparison in the following example we define the Johnstone space [12] and sketch the well-known proof of Proposition 4.3.

Example 4.4 Let $\leq$ on $\mathbb{N} \cup\{\omega\}$ be the usual linear ordering with $n \leq \omega$ for all $n \in$ $\mathbb{N} \cup\{\omega\}$. On $X:=\mathbb{N} \times(\mathbb{N} \cup\{\omega\})$ we define a binary relation $\leq_{X}$ by

$$
(i, p) \leq_{X}(j, q): \Longleftrightarrow((i=j \text { and } p \leq q) \text { or }(q=\omega \text { and } p \leq j))
$$

Then the pair $\left(X, \leq_{X}\right)$ is a dcpo. It is called the Johnstone space. Let $Y$ be the set of all Scott open subsets of $X$, and let the binary relation $\leq_{Y}$ on $Y$ be set-theoretic inclusion. By Example $3.2\left(Y, \leq_{Y}\right)$ is a complete lattice, hence, a dcpo as well. One can check that the subset $E \subseteq X \times Y$ defined by

$$
E:=\{(x, y) \in X \times Y: x \in y\}
$$

is open in the Scott topology on $\left(X \times Y, \leq_{X \times Y}\right)$ but not open in the product topology of the Scott topology on $\left(X, \leq_{X}\right)$ and the Scott topology on $\left(Y, \leq_{Y}\right)$.

For later use, we explicitly formulate one general step in the previous example.

Lemma 4.5 Let $\left(X, \leq_{X}\right)$ be a poset, and let $\left(Y, \leq_{Y}\right)$ be the complete lattice consisting of the Scott open subsets of $X$ where $\leq_{Y}$ is set-theoretic inclusion. Then the set

$$
E:=\{(x, y) \in X \times Y: x \in y\}
$$

is a Scott open subset of $X \times Y$, that is, open in the Scott topology on $\left(X \times Y, \leq_{X \times Y}\right)$. 
Proof Let $(x, y) \in E$ and $\left(x^{\prime}, y^{\prime}\right) \in X \times Y$ be a pair with $(x, y) \leq_{X \times Y}\left(x^{\prime}, y^{\prime}\right)$. Then $x \leq_{X} x^{\prime}$ and $y \leq_{Y} y^{\prime}$. Furthermore, $x \in y$. As $y$ is upwards closed, we obtain $x^{\prime} \in y$. And $y \leq_{Y} y^{\prime}$ means $y \subseteq y^{\prime}$, hence, $x^{\prime} \in y^{\prime}$, hence, $\left(x^{\prime}, y^{\prime}\right) \in E$. We have shown that $E$ is $\leq X \times Y$-upwards closed.

Now let $S \subseteq X \times Y$ be a directed subset such that $\sup _{\leq_{X \times Y}}(S)$ exists and is an element of $E$. According to Lemma 4.1(3) the suprema $\sup _{\leq_{X}}\left(\pi_{1}[S]\right)$ and $\sup _{\leq_{Y}}\left(\pi_{2}[s]\right)$ exist and satisfy $\left(\sup _{\leq_{X}}\left(\pi_{1}[S]\right), \sup _{\leq_{Y}}\left(\pi_{2}[S]\right)\right)=\sup _{\leq X \times Y}(S) \in E$. Thus, $\sup _{\leq X}\left(\pi_{1}[S]\right) \in \sup _{\leq_{Y}}\left(\pi_{2}[S]\right)=\bigcup \pi_{2}[S]$ (compare Example 3.2). We conclude that there is some $\tilde{y} \in \pi_{2}[S]$ with $\sup _{\leq X}\left(\pi_{1}[S]\right) \in \tilde{y}$. By Lemma 4.1 (2) the set $\pi_{1}[S]$ is a directed subset of $X$. As $\tilde{y}$ is a Scott open subset of $X$ we conclude that there exists some $\widehat{x} \in \pi_{1}[S] \cap \tilde{y}$. The fact $\widehat{x} \in \pi_{1}[S]$ implies that there exists some $\widehat{y} \in Y$ with $(\widehat{x}, \widehat{y}) \in S$. Analogously, the fact $\tilde{y} \in \pi_{2}[S]$ implies that there exists some $\tilde{x} \in X$ with $(\tilde{x}, \tilde{y}) \in S$. As $S$ is directed there exists some $(x, y) \in S$ with $(\widehat{x}, \widehat{y}) \leq_{X \times Y}(x, y)$ and $(\tilde{x}, \tilde{y}) \leq_{X \times Y}(x, y)$. Finally, similarly as in the proof above that $E$ is upwards closed, from $\widehat{x} \in \tilde{y}$ we conclude $x \in y$, hence, $(x, y) \in E$. This shows $S \cap E \neq \emptyset$. We have shown that $E$ is a Scott open subset of $X \times Y$.

By a modification (which is mostly a simplification) of the definition of the Johnstone space we can prove the following slight improvement of Proposition 4.3. It will be used in the proof of Theorem 7.4 in Sect. 7.

Proposition 4.6 There exist complete lattices $\left(X, \leq_{X}\right)$ and $\left(Y, \leq_{Y}\right)$ such that the following three conditions are satisfied:

1. the Scott topology on $\left(X \times Y, \leq_{X \times Y}\right)$ is strictly finer than the product topology on $X \times Y$ (of the Scott topology on $\left(X, \leq_{X}\right)$ and the Scott topology on $\left(Y, \leq_{Y}\right)$ ),

2. but the Scott topology on $X \times X$ is identical with the product topology on $X \times X$,

3. and the Scott topology on $Y \times Y$ is identical with the product topology on $Y \times Y$.

In the following example we shall define our modification $\left(X, \leq_{X}\right)$ of the Johnstone space. For $\left(Y, \leq_{Y}\right)$ we will then take the set of Scott open subsets of $X$ and the subset relation. Then we formulate and prove several lemmas that collect the important properties of $\left(X, \leq_{X}\right)$ and $\left(Y, \leq_{Y}\right)$ and from which we then deduce Proposition 4.6.

Example 4.7 Our modification of the Johnstone space is defined as follows. Let

$$
X:=\{\perp, \top\} \cup(\mathbb{N} \times \mathbb{N})
$$

where $\perp$ and $T$ are different elements that are not elements of $\mathbb{N} \times \mathbb{N}$. We define a binary relation $\leq_{X}$ on $X$ by

$$
\begin{aligned}
x \leq_{X} \quad y: \Longleftrightarrow \begin{array}{l}
x=\perp \text { or } y=\top \text { or }(\exists i \in \mathbb{N})(\exists p, q \in \mathbb{N}) \\
(x=(i, p) \text { and } y=(i, q) \text { and } p \leq q)
\end{array}
\end{aligned}
$$

for all $x, y \in X$. Compare Fig. 1 .

The reader is invited to convince himself that this poset is indeed simpler than the Johnstone space by comparing the graphical representation in Fig. 1 with the graphical representation of the Johnstone space in [7, Figure 5.2 on Page 143]. 
Fig. 1 The binary relation $\leq$ on $X:=\{\perp, \top\} \cup(\mathbb{N} \times \mathbb{N})$ defined in Example 4.7 is the reflexive and transitive closure of the binary relation given by the arrows in this figure

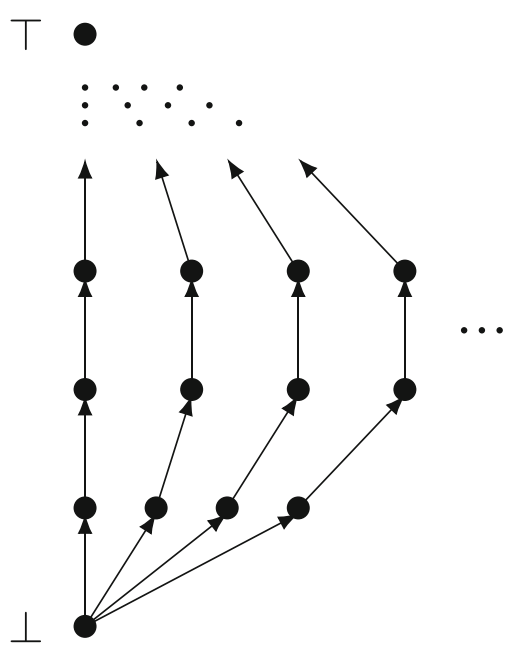

Remark 4.8 The construction in Example 4.7 was obtained by the author by slightly simplifying a similar construction in the preprint [8]. In the recent article [2] Chen, Kou, and Lyu arrive at the same construction. They also observe that it is a complete lattice and explicitly describe [2, Example 4.13] the lattice of its Scott open subsets in the same way as this is done in Lemma 4.9 (2) and 4.9 (3).

In the following lemma we show that $\left(X, \leq_{X}\right)$ is a complete lattice and give an explicit description of all Scott open subsets of $X$.

Lemma 4.9 Let $\left(X, \leq_{X}\right)$ be the pair defined in Example 4.7.

1. This pair $\left(X, \leq_{X}\right)$ is a complete lattice. In fact, the supremum of an arbitrary subset $S \subseteq X$ is given as follows:

(a) If $S=\emptyset$ or $S=\{\perp\}$ then $\sup (S)=\perp$.

(b) If $S$ is finite but neither empty nor equal to $\{\perp\}$ and if there exists a number $i \in \mathbb{N}$ with $S \subseteq\{\perp\} \cup(\{i\} \times \mathbb{N})$ then $\sup (S)=(i, \max \{p:(i, p) \in S\})$.

(c) In all other cases $\sup (S)=\top$.

2. For any total function $f: \mathbb{N} \rightarrow \mathbb{N}$ the set

$$
U_{f}:=\{\top\} \cup\{(i, p) \in \mathbb{N} \times \mathbb{N}: p \geq f(i)\}
$$

is a Scott open subset of $X$.

3. For every Scott open subset $V \subseteq X$ with $V \notin\{\emptyset, X\}$ there exists a function $f: \mathbb{N} \rightarrow \mathbb{N}$ with $V=U_{f}$.

Proof 1. It is straightforward to check that $\leq_{X}$ is a partial order. It is clear that $\sup (\emptyset)=\perp$ and $\sup (\{\perp\})=\perp$ as well. And if, for some $i \in \mathbb{N}$, the set $S$ is a finite subset of $\{\perp\} \cup\{i\} \times \mathbb{N}$ with $S \cap(\{i\} \times \mathbb{N}) \neq \varnothing$ then it is clear as well that $\sup (S)=(i, \max \{p:(i, p) \in S\})$. Let us consider the third case. If there are $i, j, p, q \in \mathbb{N}$ with $i \neq j$ and $(i, p),(j, q) \in S$ then clearly $\sup (S)=\top$. If $S$ is 
infinite then also $\sup (S)=\top$. Thus, $\sup (S)$ exists for every subset $S \subseteq X$. Hence, $\left(X, \leq_{X}\right)$ is a a complete lattice.

2. Let $f: \mathbb{N} \rightarrow \mathbb{N}$ be a total function. It is clear that $U_{f}$ is upwards closed. Let $S \subseteq X$ be a directed subset such that $\sup (S)$ exists and $\sup (S) \in U_{f}$. We wish to show that there exists an element $s \in S \cap U_{f}$. This is clear if $\top \in S$ because then $\sup (S)=\top \in U_{f}$, hence, $\top \in S \cap U_{f}$. So, let us assume that $\top \notin S$. Can there exist numbers $i, j, p, q \in \mathbb{N}$ with $i \neq j$ and $(i, p) \in S$ and $(j, q) \in S$ ? No. As $S$ is directed, in that case there would have to exist some $s \in S$ with $(i, p) \leq_{X} s$ and $(j, q) \leq_{X} s$. But the only element $s$ of $X$ that satisfies $(i, p) \leq_{X} s$ and $(j, q) \leq_{X} s$ is the element $s=\top$. Hence, we would get $\top \in S$. So, we can conclude that there exists some $i \in \mathbb{N}$ such that $S \subseteq\{\perp\} \cup(\{i\} \times \mathbb{N})$. If $S$ is infinite then there exists some $p \in \mathbb{N}$ such that $p \geq f(i)$ and $(i, p) \in S$. As we also have $(i, p) \in U_{f}$, we conclude $(i, p) \in S \cap U_{f}$. Finally, let us consider the case that $S$ is finite and $S \subseteq\{\perp\} \cup(\{i\} \times \mathbb{N})$. As $\perp$ is not an element of $U_{f}$, the set $S$ can neither be empty nor be equal to $\{\perp\}$ because in both cases one would arrive at $\sup (S)=\perp$. Thus, we obtain $\sup (S)=(i, \max \{p:(i, p) \in S\})$. But in this case we have not only $\sup (S) \in U_{f}$ but also $\sup (S) \in S$.

3. Let $V \subseteq X$ be a Scott open subset of $X$ with $V \neq \varnothing$ and $V \neq X$. Then $\top \in V$ (otherwise $V=\emptyset$ ) and $\perp \notin V$ (otherwise $V=X$ ). For any $i \in \mathbb{N}$ the set $\{i\} \times \mathbb{N}$ is a directed subset of $X$ with $\sup (\{i\} \times \mathbb{N})=\top \in V$. Hence, for any $i \in \mathbb{N}$ there exists some $p \in \mathbb{N}$ with $(i, p) \in V$. Therefore, we can define a function $f: \mathbb{N} \rightarrow \mathbb{N}$ by

$$
f(i):=\min \{p \in \mathbb{N}:(i, p) \in V\}
$$

for $i \in \mathbb{N}$, and see that $V=U_{f}$.

Lemma 4.10 Let $\left(X, \leq_{X}\right)$ be the complete lattice defined in Example 4.7 (see Lemma $4.9(1))$, and let $\left(Y, \leq_{Y}\right)$ be the complete lattice where $Y$ is the set of all Scott open subsets of $X$ and $\leq_{Y}$ is set-theoretic inclusion (see Example 3.2). Then the subset

$$
E:=\{(x, y) \in X \times Y: x \in y\}
$$

is not open with respect to the product topology on $X \times Y$.

Proof First, let us choose an element of $E$ as follows. Let $\tilde{0}: \mathbb{N} \rightarrow \mathbb{N}$ be the constant number function with $\tilde{0}(n)=0$ for all $n \in \mathbb{N}$. Then, according to Lemma 4.9 (2), the set $y:=U_{\tilde{0}}=X \backslash\{\perp\}$ is a Scott open subset of $X$. The element $x:=(0,0) \in X$ is an element of $y$. Thus, $(x, y) \in E$.

Now, for the sake of a contradiction, let us assume that $E$ is open in the product topology. Then there exist Scott open subsets $U \subseteq X$ and $\mathcal{V} \subseteq Y$ with $(x, y) \in U \times \mathcal{V}$ and $U \times \mathcal{V} \subseteq E$. The fact $(x, y) \in U \times \mathcal{V}$ implies $U \neq \emptyset$. Can $U$ be equal to $X$ ? No, because otherwise we would have $X \times \mathcal{V} \subseteq E$, hence $x \in V$, for all $x \in X$ and $V \in \mathcal{V}$. This would imply $X \subseteq V$ for all $V \in \mathcal{V}$, hence, $\mathcal{V}=\{X\}$ in contradiction to $X \backslash\{\perp\}=y \in \mathcal{V}$. So, we conclude that $U \neq \emptyset$ and $U \neq X$. According to Lemma 4.9 
(3) there exists some function $f: \mathbb{N} \rightarrow \mathbb{N}$ such that $U=U_{f}$. For $i \in \mathbb{N}$ we define $g_{i}: \mathbb{N} \rightarrow \mathbb{N}$ by

$$
g_{i}(m):= \begin{cases}0 & \text { if } m<i \\ f(m)+1 & \text { if } m \geq i\end{cases}
$$

Then the sequence $\left(U_{g_{i}}\right)_{i \in \mathbb{N}}$ is an increasing sequence of Scott open subsets of $X$ with

$$
\sup \left(\left\{U_{g_{i}}: i \in \mathbb{N}\right\}\right)=\bigcup_{i \in \mathbb{N}} U_{g_{i}}=X \backslash\{\perp\}=U_{\tilde{0}}=y \in \mathcal{V}
$$

As $\mathcal{V}$ is a Scott open subset of $Y$, there must exist some $i \in \mathbb{N}$ with $U_{g_{i}} \in \mathcal{V}$. As $U \times \mathcal{V} \subseteq E$, we obtain $\left(x, U_{g_{i}}\right) \in E$, for all $x \in U$, hence $x \in U_{g_{i}}$, for all $x \in U$, hence, $U \subseteq U_{g_{i}}$. But that is false!

Remark 4.11 It is instructive to discuss the previous lemma in the context of some further domain-theoretic notions. Let $(Z, \leq)$ be a poset.

- For $x, y \in Z$ we write $x \ll y$ and say that $x$ is way-below $y$ if for every directed set $S \subseteq Z$ such that $\sup (S)$ exists and satisfies $y \leq \sup (S)$ there exists an element $s \in S$ with $x \leq s$.

- The poset $(Z, \leq)$ is called continuous if, for every $z \in Z$, the set $\{x \in Z: x \ll z\}$ has the following properties: (1) it is directed, (2) its supremum exists, (3) its supremum is equal to $z$.

- The poset $(Z, \leq)$ is called core-compact if the poset $(\sigma(Z, \leq), \subseteq)$ is a continuous poset.

In the following, as in Lemma 4.10, we consider the complete lattice $\left(X, \leq_{X}\right)$ defined in Example 4.7 and the complete lattice $\left(Y, \leq_{Y}\right):=\left(\sigma\left(X, \leq_{X}\right), \subseteq\right)$. First, it is obvious that $\left(X, \leq_{X}\right)$ is not continuous (because $\left.\{x \in X: x \ll \top\}=\{\perp\}\right)$. Secondly, it is well known that the assertion proved in the previous lemma (that the set $E:=$ $\{(x, y) \in X \times Y: x \in y\}$ is not open with respect to the product topology on $X \times Y)$ is equivalent to the assertion that $\left(Y, \leq_{Y}\right)$ is not continuous, thus, to the assertion that $\left(X, \leq_{X}\right)$ is not core-compact (indeed, the conditions (4) and (6) in [6, Theorem II-4.10] are equivalent). Thus, neither $\left(X, \leq_{X}\right)$ nor $\left(Y, \leq_{Y}\right)$ are continuous lattices.

Note also that, if $\left(\sigma\left(X, \leq_{X}\right), \subseteq\right)$ were continuous then, due to [6, Theorem II-4.13], this would imply that for any dcpo $\left(Z, \leq_{Z}\right)$ the product topology on $X \times Z$ would coincide with the Scott topology on $X \times Z$. But, we have just seen that $\left(\sigma\left(X, \leq_{X}\right), \subseteq\right)$ is not continuous. And, indeed, Lemmas 4.5 and 4.10 show that the product topology on $X \times Y$ (where again $\left(Y, \leq_{Y}\right):=\left(\sigma\left(X, \leq_{X}\right), \subseteq\right)$ ) does not coincide with the Scott topology on $X \times Y$. Nevertheless, the Scott topology on $X \times X$ coincides with the product topology on $X \times X$.

Lemma 4.12 Let $\left(X, \leq_{X}\right)$ be the complete lattice defined in Example 4.7 (see Lemma 4.9(1)). Then the Scott topology on $X \times X$ coincides with the product topology on $X \times X$. 
Proof By Lemma 4.2 it is sufficient to show that any Scott open subset of $X \times X$ is open in the product topology on $X \times X$. So, let us consider some Scott open subset $W$ of $X \times X$. If $W$ is empty then it is open in the product topology. So, let us assume that $W$ is not empty. Let us fix some element $\left(x_{1}, x_{2}\right) \in W$. It is sufficient to show that there exist Scott open subsets $U, V \subseteq X$ with $x_{1} \in U$, with $x_{2} \in V$, and with $U \times V \subseteq W$. There are three cases for $x_{1}$ : (1) $x_{1}=\perp$, (2) $x_{1} \in \mathbb{N} \times \mathbb{N}$, (3) $x_{1}=\top$, similarly for $x_{2}$. So, we have to consider nine cases.

Case $1 x_{1}=x_{2}=\perp$. Then, as $W$ is upwards closed, it must be equal to $X \times X$. Hence, it is open in the product topology.

Case $2 x_{1}=\perp$ and $x_{2}=\top$. Similarly as in the proof of Lemma 4.9 (3) one shows that there exists a function $f: \mathbb{N} \rightarrow \mathbb{N}$ with $X \times U_{f} \subseteq W$. Let us show this in detail. For every $i \in \mathbb{N}$ the set $S_{i}:=\{\perp\} \times(\{i\} \times \mathbb{N})$ is a directed subset of $X \times X$ with $\sup (S)=(\perp, \top)=\left(x_{1}, x_{2}\right) \in W$. We conclude that $S_{i} \cap W \neq \emptyset$. We define $f: \mathbb{N} \rightarrow \mathbb{N}$ by $f(i):=\min \{p:(\perp,(i, p)) \in W\}$. As $W$ is upwards closed, $X \times U_{f} \subseteq W$. Finally, we note that $(\perp, \top) \in X \times U_{f}$.

Case $3 x_{1}=\perp$ and $x_{2} \in \mathbb{N} \times \mathbb{N}$. As $\left(\perp, x_{2}\right) \leq_{X \times X}(\perp, \top)$ and $W$ is upwards closed we see $(\perp, \top) \in W$. As in Case 2 , we define $f: \mathbb{N} \rightarrow \mathbb{N}$ by $f(i):=$ $\min \{p:(\perp,(i, p)) \in W\}$, for all $i \in \mathbb{N}$. As $W$ is upwards closed, $X \times U_{f} \subseteq W$. Let $j, q$ be the numbers with $x_{2}=(j, q)$. By definition of $f, f(j) \leq q$, hence, $\left(x_{1}, x_{2}\right)=(\perp,(j, q)) \in X \times U_{f}$.

Case $4 x_{1}=\top$ and $x_{2}=\perp$. This case is symmetric to Case 2 and is treated in the same way.

Case $5 x_{1} \in \mathbb{N} \times \mathbb{N}$ and $x_{2}=\perp$. This case is symmetric to Case 3 and is treated in the same way.

Case $6 x_{1} \in \mathbb{N} \times \mathbb{N}$ and $x_{2} \in \mathbb{N} \times \mathbb{N}$. We shall define functions $f: \mathbb{N} \rightarrow \mathbb{N}$ and $g: \mathbb{N} \rightarrow \mathbb{N}$ with $x_{1} \in U_{f}, x_{2} \in U_{g}$, and $U_{f} \times U_{g} \subseteq W$. Let $i_{1}, p_{1}, i_{2}, p_{2}$ be the numbers with $x_{1}=\left(i_{1}, p_{1}\right)$ and $x_{2}=\left(i_{2}, p_{2}\right)$. We define the functions $f$ and $g$ step by step and, additionally, in parallel two increasing sequences of finite sets $N_{1} \subseteq \mathbb{N}$ and $N_{2} \subseteq \mathbb{N}$ (containing the numbers on which $f$ and $g$ have been defined already) as follows. In the first step we define

$$
\begin{aligned}
f\left(i_{1}\right) & :=p_{1}, \\
N_{1} & :=\left\{i_{1}\right\}, \\
g\left(i_{2}\right) & :=p_{2}, \\
N_{2} & :=\left\{i_{2}\right\} .
\end{aligned}
$$

Then we repeat the following infinitely often:

$$
\begin{aligned}
j_{1} & :=\min \left(\mathbb{N} \backslash N_{1}\right), \\
f\left(j_{1}\right) & :=\min \left\{q \in \mathbb{N}:\left(\forall k_{2} \in N_{2}\right)\left(\left(j_{1}, q\right),\left(k_{2}, g\left(k_{2}\right)\right)\right) \in W\right\}, \\
N_{1} & :=N_{1} \cup\left\{j_{1}\right\},
\end{aligned}
$$




$$
\begin{aligned}
j_{2} & :=\min \left(\mathbb{N} \backslash N_{2}\right), \\
g\left(j_{2}\right) & :=\min \left\{q \in \mathbb{N}:\left(\forall k_{1} \in N_{1}\right)\left(\left(k_{1}, f\left(k_{1}\right)\right),\left(j_{2}, q\right)\right) \in W\right\}, \\
N_{2} & :=N_{2} \cup\left\{j_{2}\right\} .
\end{aligned}
$$

Let us show that these definitions make sense and define functions $f$ and $g$ with the desired properties. By induction we show that after each step we have $\left(\left(k_{1}, f\left(k_{1}\right)\right),\left(k_{2}, g\left(k_{2}\right)\right)\right) \in W$, for all $k_{1} \in N_{1}$ and all $k_{2} \in N_{2}$. It is clear that this is true after the first step, that is, for $N_{1}=\left\{i_{1}\right\}$ and $N_{2}=\left\{i_{2}\right\}$. Let us assume by induction that this is true after some step. We show that it is true after the next step as well. Note that for every $k_{2} \in N_{2}$ and $j_{1}:=\min \left(\mathbb{N} \backslash N_{1}\right)$ the set $\left(\left\{j_{1}\right\} \times \mathbb{N}\right) \times\left\{\left(k_{2}, g\left(k_{2}\right)\right)\right\}$ is a directed subset of $X \times X$ whose supremum ( $\left.丁,\left(k_{2}, g\left(k_{2}\right)\right)\right)$ is an element of $W$ (remember that $\left(\left(i_{1}, f\left(i_{1}\right)\right),\left(k_{2}, g\left(k_{2}\right)\right)\right) \in W$ by induction hypothesis and $\left.\left(\left(i_{1}, f\left(i_{1}\right)\right),\left(k_{2}, g\left(k_{2}\right)\right)\right) \leq X \times X \quad\left(\top,\left(k_{2}, g\left(k_{2}\right)\right)\right)\right)$. Thus, there exists some $q \in \mathbb{N}$ with $\left(\left(j_{1}, q\right),\left(k_{2}, g\left(k_{2}\right)\right)\right) \in W$. As $W$ is upwards closed and $N_{2}$ is finite in every step of the definition of $f, f\left(j_{1}\right)$ is well-defined for $j_{1}=\min \left(\mathbb{N} \backslash N_{1}\right)$, and we obtain $\left(\left(j_{1}, f\left(j_{1}\right)\right),\left(k_{2}, g\left(k_{2}\right)\right)\right) \in W$, for this $j_{1}$ and all $k_{2} \in N_{2}$. The same argument applies to the definition of $g\left(j_{2}\right)$. This ends the proof by induction. Thus, by the steps above we have defined two functions $f: \mathbb{N} \rightarrow \mathbb{N}$ and $g: \mathbb{N} \rightarrow \mathbb{N}$ such that $\left(\left(k_{1}, f\left(k_{1}\right)\right),\left(k_{2}, g\left(k_{2}\right)\right)\right) \in W$, for all $k_{1} \in \mathbb{N}$ and all $k_{2} \in \mathbb{N}$. This shows $U_{f} \times U_{g} \subseteq W$. Finally, it is clear that $x_{1}=\left(i_{1}, p_{1}\right) \in U_{f}$ and that $x_{2}=\left(i_{2}, p_{2}\right) \in U_{g}$. This ends the treatment of Case 6 .

Case $7 x_{1} \in \mathbb{N} \times \mathbb{N}$ and $x_{2}=\top$. The set $\left\{x_{1}\right\} \times(\{0\} \times \mathbb{N})$ is a directed subset of $X \times X$ whose supremum $\left(x_{1}, \top\right)=\left(x_{1}, x_{2}\right)$ is an element of $W$. Hence, there exists some number $p$ with $\left(x_{1},(0, p)\right) \in W$. By Case 6 there exist functions $f: \mathbb{N} \rightarrow \mathbb{N}$ and $g: \mathbb{N} \rightarrow \mathbb{N}$ with $x_{1} \in U_{f},(0, p) \in U_{g}$ and $U_{f} \times U_{g} \subseteq W$. As $\top \in U_{g}$ is clear, this ends the treatment of Case 7 .

Case $8 x_{1}=\top$ and $x_{2} \in \mathbb{N} \times \mathbb{N}$. This case is symmetric to Case 7 and is treated in the same way.

Case $9 x_{1}=x_{2}=\top$. The set $(\{0\} \times \mathbb{N}) \times\{\top\}$ is a directed subset of $X \times X$ whose supremum $(T, T)=\left(x_{1}, x_{2}\right)$ is an element of $W$. Hence, there exists some number $p$ with $((0, p), \top) \in W$. By Case 7 there exist functions $f: \mathbb{N} \rightarrow \mathbb{N}$ and $g: \mathbb{N} \rightarrow \mathbb{N}$ with $(0, p) \in U_{f}, \top \in U_{g}$ and $U_{f} \times U_{g} \subseteq W$. As $\top \in U_{f}$ is clear, this ends the treatment of Case 9.

In the following let $\left(X, \leq_{X}\right)$ be the complete lattice defined in Example 4.7, and let $\left(Y, \leq_{Y}\right)$ be the complete lattice consisting of the set $Y$ of Scott open subsets of $X$ and the subset relation $\leq_{Y}:=\subseteq$ on $Y$. A partial function $f: \subseteq \mathbb{N} \rightarrow \mathbb{N}$ with finite domain is any function $f: F \rightarrow \mathbb{N}$ where $F \subseteq \mathbb{N}$ is a finite subset of $\mathbb{N}$. In this case $F$ is called the domain of $f$, and we write $\operatorname{dom}(f):=F$. For any partial function $g: \subseteq \mathbb{N} \rightarrow \mathbb{N}$ with finite domain let

$$
\begin{gathered}
\mathcal{V}_{g}:=\{X\} \cup\left\{U_{f}: f: \mathbb{N} \rightarrow \mathbb{N}\right. \text { is a total function with } \\
(\forall i \in \operatorname{dom}(g)) f(i) \leq g(i)\} .
\end{gathered}
$$


In the following lemma we identify some Scott open subsets of $Y$.

Lemma 4.13 1. The set $\{X\}$ is a Scott open subset of $Y$.

2. For any partial function $g: \subseteq \mathbb{N} \rightarrow \mathbb{N}$ with finite domain the set $\mathcal{V}_{g}$ is a Scott open subset of $Y$.

Proof It is clear that the set $\{X\}$ is upwards closed. Let $\mathcal{S} \subseteq Y$ be a directed subset with $\sup (\mathcal{S}) \in\{X\}$. Then $\sup (\mathcal{S})=X$, in particular $\perp \in \sup (\mathcal{S})$. $\operatorname{As} \sup (\mathcal{S})$ is the union of all sets that are elements of $\mathcal{S}$ there must exist some $U \in \mathcal{S}$ with $\perp \in U$. As $U$ is a Scott open subset of $X$ this implies $U=X$, hence, $X \in \mathcal{S}$. We have shown that $\{X\}$ is a Scott open subset of $Y$.

Now let $g: \subseteq \mathbb{N} \rightarrow \mathbb{N}$ be a partial function with finite domain. We wish to show that $\mathcal{V}_{g}$ is a Scott open subset of $Y$. If the domain of $g$ is empty then $\mathcal{V}_{g}$ is equal to $Y \backslash\{\emptyset\}$, and this is obviously a Scott open subset of $Y$. Let us assume that $g$ is not empty. Let $i_{0}, \ldots, i_{e-1}$ be the elements of $\operatorname{dom}(g)$, and let $g_{j}$ be the restriction of $g$ to $\left\{i_{j}\right\}$, for $j=0, \ldots, e-1$. Then

$$
\mathcal{V}_{g}=\bigcap_{j=0}^{e-1} \mathcal{V}_{g_{j}}
$$

Therefore, we can assume without loss of generality that $\operatorname{dom}(g)$ contains exactly one number. Let $i_{0}$ be this number, and let $p_{0}:=g\left(i_{0}\right)$. Then

$$
\mathcal{V}_{g}=\{X\} \cup\left\{U_{f}: f: \mathbb{N} \rightarrow \mathbb{N} \text { is a total function with } f\left(i_{0}\right) \leq p_{0}\right\}
$$

It is clear that this set is upwards closed. Let $\mathcal{S} \subseteq Y$ be a directed subset with $\sup (\mathcal{S}) \in$ $\mathcal{V}_{g}$. If $\sup (\mathcal{S})=X$ then we can conclude as above in the proof of the first assertion of this lemma that $X \in \mathcal{S}$. So, in this case we have $S \cap \mathcal{V}_{g} \neq \emptyset$. Let us assume that $\sup (\mathcal{S})=U_{f}$ for some total function $f: \mathbb{N} \rightarrow \mathbb{N}$ with $U_{f} \in \mathcal{V}_{g}$, hence, with $f\left(i_{0}\right) \leq p_{0}$, hence, with $\left(i_{0}, p_{0}\right) \in U_{f}$. As $\sup (\mathcal{S})$ is the union of all sets that are elements of $\mathcal{S}$ there must exist some $U \in \mathcal{S}$ with $\left(i_{0}, p_{0}\right) \in U$. As $U=X$ is impossible (this would imply $\sup (\mathcal{S})=X$ ) we conclude that there is some function $h: \mathbb{N} \rightarrow \mathbb{N}$ with $U=U_{h}$ and $h\left(i_{0}\right) \leq p_{0}$. We obtain $U \in \mathcal{S} \cap \mathcal{V}_{g}$, hence, $\mathcal{S} \cap \mathcal{V}_{g} \neq \emptyset$.

Actually, the Scott open subsets of $Y$ identified in the previous lemma already form a base of the Scott topology of $Y$. Thus, the Scott topology of $\left(Y, \leq_{Y}\right)$ has a countable base.

Lemma 4.14 The set

$$
\begin{aligned}
\mathcal{B}:=\{\{X\}, Y\} \cup\left\{\mathcal{V}_{g}:\right. & g: \subseteq \mathbb{N} \rightarrow \mathbb{N} \text { is a partial function with finite } \\
& \text { domain, and }(\exists n \in \mathbb{N}) \operatorname{dom}(g)=\{0, \ldots, n-1\}\}
\end{aligned}
$$

is a base of the topology of $\left(Y, \leq_{Y}\right)$.

Proof According to Lemma 4.13 all elements of the set $\mathcal{B}$ are Scott open subsets of $\left(Y, \leq_{Y}\right)$. Let $\mathcal{Z} \subseteq Y$ be a Scott open subset of $Y$. Let $U \subseteq X$ be a Scott open subset of 
$X$ with $U \in \mathcal{Z}$. It is sufficient to show that there exists a set $\mathcal{V} \in \mathcal{B}$ with $U \in \mathcal{V} \subseteq \mathcal{Z}$. In the case $U=\emptyset$ we obtain $\mathcal{Z}=Y$ and can use $\mathcal{V}:=Y$. In the case $U=X$ we can simply use $\mathcal{V}:=\{X\}$. In the following we consider the case $U \notin\{\emptyset, X\}$. Then, according to Lemma 4.9 (3) there exists a function $f: \mathbb{N} \rightarrow \mathbb{N}$ with $U=U_{f}$. We claim that there exists a number $k \in \mathbb{N}$ with $\mathcal{V}_{f \nmid k} \subseteq \mathcal{Z}$. For the sake of a contradiction, let us assume that this is not the case. Then there exists a sequence $V: \mathbb{N} \rightarrow Y$ such that, for all $k \in \mathbb{N}, V(k) \in \mathcal{V}_{f \nmid k}$ and $V(k) \notin \mathcal{Z}$. For every $k$ either $V(k)=X$ or there exists a function $g_{k}: \mathbb{N} \rightarrow \mathbb{N}$ with $V(k)=U_{g_{k}}$. In the first case we define $g_{k}:=f$ and observe $U_{g_{k}} \subseteq X=V(k)$ and $U_{g_{k}} \in \mathcal{V}_{f \nmid k}$. So, for every $k \in \mathbb{N}$ there exists a function $g_{k}: \mathbb{N} \rightarrow \mathbb{N}$ with $U_{g_{k}} \subseteq V(k)$ and $U_{g_{k}} \in \mathcal{V}_{f \nmid k}$. We are going to construct a sequence $W: \mathbb{N} \rightarrow Y$ of Scott open subsets of $X$ such that

$$
\bigcup_{k \in \mathbb{N}} W(k)=U_{f} \text { and }(\forall k \in \mathbb{N})\left(W(k) \subseteq U_{g_{k}} \text { and } W(k) \subseteq W(k+1)\right)
$$

In order to construct such a sequence, we define a function $g: \mathbb{N} \rightarrow \mathbb{N}$ by

$$
g(j):=\max \left(\{f(j)\} \cup\left\{g_{k}(j): k \leq j\right\}\right),
$$

for $j \in \mathbb{N}$. It is clear that $f(j) \leq g(j)$, for all $j$. Furthermore, for all $k, j \in \mathbb{N}$, $g_{k}(j) \leq g(j)$. Indeed, this is clear for $k \leq j$ by definition of $g$, and for $j<k$ we observe that $U_{g_{k}} \in \mathcal{V}_{f\lceil k}$ implies $g_{k}(j) \leq f(j)$, hence, $g_{k}(j) \leq f(j) \leq g(j)$. For $k \in \mathbb{N}$ we define a function $h_{k}: \mathbb{N} \rightarrow \mathbb{N}$ by

$$
h_{k}(j):= \begin{cases}f(j) & \text { if } j<k \\ g(j) & \text { if } k \leq j\end{cases}
$$

We observe that for all $k, j \in \mathbb{N}$

$$
f(j) \leq h_{k+1}(j) \leq h_{k}(j)
$$

and

$$
g_{k}(j) \leq h_{k}(j)
$$

(indeed, for $j<k$ we have already seen $g_{k}(j) \leq f(j)=h_{k}(j)$, and for $k \leq j$ we see $\left.g_{k}(j) \leq g(j)=h_{k}(j)\right)$. Furthermore, $\lim _{k \rightarrow \infty} h_{k}(j)=f(j)$, for all $j$. Thus, the sequence $W: \mathbb{N} \rightarrow Y$ defined by $W(k):=U_{h_{k}}$, for $k \in \mathbb{N}$, has the desired property (1). The set $\{W(k): k \in \mathbb{N}\}$ is a directed subset of $Y$ with $\sup \{W(k): k \in$ $\mathbb{N}\}=U_{f}=U$. As $\mathcal{Z}$ is a Scott open subset of $Y$ and $U \in \mathcal{Z}$ there exists a number $k$ with $W(k) \in \mathcal{Z}$, hence, as $\mathcal{Z}$ is upwards closed and $W(k) \subseteq U_{g_{k}} \subseteq V(k)$, with $V(k) \in \mathcal{Z}$. Contradiction. We have shown that there exists a number $k \in \mathbb{N}$ with $\mathcal{V}_{f \nmid k} \subseteq W$. Note that $U=U_{f} \in \mathcal{V}_{f\lceil k}$.

A topological space $(X, \tau)$ is called first-countable if for every point $x \in X$ there exists a sequence $B: \mathbb{N} \rightarrow \tau$ of open sets such that, for all $k \in \mathbb{N}, x \in B(k)$ and, for 
any open subset $U \subseteq X$ with $x \in U$ there exists a $k \in \mathbb{N}$ with $B(k) \subseteq U$. Matthew de Brecht made the following observation, explained in detail in the blog of Jean Goubault-Larrecq [3].

Proposition 4.15 [de Brecht [3]] Let $\left(Z_{1}, \leq_{1}\right)$ and $\left(Z_{2}, \leq_{2}\right)$ be posets such that the Scott spaces $\left(Z_{1}, \sigma\left(Z_{1}, \leq_{1}\right)\right)$ and $\left(Z_{2}, \sigma\left(Z_{2}, \leq_{2}\right)\right)$ are first-countable. Then the $S$ cott topology on $Z_{1} \times Z_{2}$ coincides with the product topology on $Z_{1} \times Z_{2}$.

Corollary 4.16 Let $\left(X, \leq_{X}\right)$ be the complete lattice defined in Example 4.7 (see Lemma $4.9(1))$, and let $\left(Y, \leq_{Y}\right)$ be the complete lattice where $Y$ is the set of all Scott open subsets of $X$ and $\leq_{Y}$ is set-theoretic inclusion (see Example 3.2). Then the Scott topology on $Y \times Y$ coincides with the product topology on $Y \times Y$.

Proof In Lemma 4.13 we have seen that the space $\left(Y, \sigma\left(Y, \leq_{Y}\right)\right)$ has a countable base. In particular, it is first-countable. By Proposition 4.15 the Scott topology on $Y \times Y$ coincides with the product topology on $Y \times Y$.

Proof of Proposition 4.6. Let $\left(X, \leq_{X}\right)$ be the pair defined in Example 4.7. According to Lemma $4.9(1)\left(X, \leq_{X}\right)$ is a complete lattice. Let $\left(Y, \leq_{Y}\right)$ be the pair consisting of the set $Y$ of all Scott open subsets of $X$ and set-theoretic inclusion $\leq_{Y}:=\subseteq$ on $Y$. By Example $3.2\left(Y, \leq_{Y}\right)$ is a complete lattice. According to Lemma 4.2 the Scott topology on $X \times Y$ is at least as fine as the product topology on $X \times Y$. The subset $E:=\{(x, y) \in X \times Y: x \in y\}$ of $X \times Y$ is Scott open according to Lemma 4.5, but not open with the respect to the product topology, according to Lemma 4.10. Hence, the Scott topology on $X \times Y$ is strictly finer than the product topology on $X \times Y$. By Lemma 4.12 the Scott topology on $X \times X$ coincides with the product topology on $X \times X$, and by Corollary 4.16 the Scott topology on $Y \times Y$ coincides with the product topology on $Y \times Y$.

\section{Elementary properties of the binary supremum function}

Let $X$ be a set. If $\leq$ is a partial order on $X$ such that $(X, \leq)$ is a sup semilattice then we define the binary supremum function $\sup _{\leq}^{(2)}: X \times X \rightarrow X$ on $X$ by

$$
\sup _{\leq}^{(2)}(x, y):=\sup (\{x, y\})
$$

for $x, y \in X$. If the relation $\leq$ is clear from the context then instead of $\sup _{\leq}^{(2)}$ we simply write sup ${ }^{(2)}$. For completeness sake let us mention the following well known algebraic characterisation of the binary supremum function. We call a function $f: X \times X \rightarrow X$

- idempotent if $(\forall x \in X) f(x, x)=x$,

- commutative if $(\forall x, y \in X) f(x, y)=f(y, x)$,

- associative if $(\forall x, y, z \in X) f(f(x, y), z)=f(x, f(y, z))$.

For a function $f: X \times X \rightarrow X$ let us define a binary relation $\leq{ }_{f} \subseteq X \times X$ by

$$
x \leq_{f} y: \Longleftrightarrow y=f(x, y)
$$


for $x, y \in X$.

Lemma 5.1 Let $X$ be a nonempty set.

1. If $\leq i$ s a partial order on $X$ such that $(X, \leq)$ is a sup semilattice then the function $f:=\sup _{\leq}^{(2)}$ is idempotent, commutative, and associative, and the relation $\leq_{f}$ is identical with $\leq$.

2. If $f: X \times X \rightarrow X$ is an idempotent, commutative, and associative function then the binary relation $\leq_{f}$ on $X$ is a partial order such that $\left(X, \leq_{f}\right)$ is a sup semilattice, and $f$ is the binary supremum function of this sup semilattice.

This is well-known, and the proof is straightforward. We omit it. This lemma shows that the transition from a sup semilattice $(X, \leq)$ (an order-theoretic object) to the pair $\left(X, \sup _{\leq}^{(2)}\right)$ consisting of a set $X$ and a function with the three properties above (an algebraic object) on the one hand and the transition from a pair $(X, f)$ consisting of a set $X$ and a function $f$ with the three properties above (an algebraic object) to the sup semilattice $\left(X, \leq_{f}\right)$ (an order-theoretic object) on the other hand are inverse to each other. In this article we work with the order-theoretic description.

Let us have a first look at topological properties of the binary supremum function on a sup semilattice. Let $\left(X, \tau_{X}\right)$ and $\left(Y, \tau_{Y}\right)$ be topological spaces. A function $f$ : $X \rightarrow Y$ is called

- continuous if for every open subset $V \subseteq Y$ its preimage $f^{-1}[V]=\{x \in$ $X: f(x) \in V\}$ is an open subset of $X$,

- open if for every open subset $U \subseteq X$ its image $f[U]=\{y \in Y:(\exists x \in U) y=$ $f(x)\}$ is an open subset of $Y$.

Often the following fundamental characterization of Scott continuous functions between partial orders is useful.

Lemma 5.2 (see, e.g., [7, Proposiation. 4.3.5]) Let $\left(X, \leq_{X}\right)$ and $\left(Y, \leq_{Y}\right)$ be posets. For a function $f: X \rightarrow Y$ the following two conditions are equivalent.

1. $f$ is Scott continuous, that is, continuous with respect to the Scott topology on $X$ and the Scott topology on $Y$.

2. $f$ is monotone (that is, $\left(\forall x, x^{\prime} \in X\right)\left(x \leq_{X} x^{\prime} \Rightarrow f(x) \leq_{Y} f\left(x^{\prime}\right)\right)$ and, if $S \subseteq X$ is a directed set whose supremum $\sup (S)$ exists, then $\sup (f[S])$ exists as well and satisfies $\sup (f[S])=f(\sup (S))$.

Proposition 5.3 Let $(X, \leq)$ be a sup semilattice. Then the function $\sup ^{(2)}: X \times X \rightarrow X$ is continuous and open with respect to the Scott topology on $X \times X$ (on the left hand side) and the Scott topology on $X$ (on the right hand side).

The continuity of $\sup ^{(2)}$ is well known [13, p. 26]. It seems that the openness of $\sup ^{(2)}$ has not been stated so far.

Proof For completeness sake, we prove the continuity of $\sup ^{(2)}$. We use Lemma 5.2. It is obvious that $\sup ^{(2)}$ is monotone. Let $S \subseteq X \times X$ be a directed set such that $\sup (S)$ exists. We have to show that $\sup ^{(2)}(\sup (S))$ is a supremum of $\sup ^{(2)}[S]$. For any 
$\left(x, x^{\prime}\right) \in S$ we have $\left(x, x^{\prime}\right) \leq X \times X \sup (S)$, hence, due to the monotonicity of $\sup ^{(2)}$, also $\sup ^{(2)}\left(x, x^{\prime}\right) \leq \sup ^{(2)}(\sup (S))$. This shows that $\sup ^{(2)}(\sup (S))$ is an upper bound of $\sup ^{(2)}[S]$. Now let $y \in X$ be an upper bound of $\sup ^{(2)}[S]$. Then for all $\left(x, x^{\prime}\right) \in S$ we have $\sup ^{(2)}\left(x, x^{\prime}\right) \leq y$, hence, $x \leq y$ and $x^{\prime} \leq y$, hence, $\left(x, x^{\prime}\right) \leq X \times X \quad(y, y)$. This implies $\sup (S) \leq_{X \times X}(y, y)$ and, due to the monotonicity of $\sup ^{(2)}, \sup ^{(2)}(\sup (S)) \leq$ $\sup ^{(2)}(y, y)=y$. Thus, $\sup ^{(2)}(\sup (S))$ is indeed a supremum of $\sup ^{(2)}[S]$.

For the openness of $\sup ^{(2)}$ let us consider a Scott open subset $U \subseteq X \times X$. We wish to show that $\sup ^{(2)}[U]$ is a Scott open subset of $X$. First we show that $\sup ^{(2)}[U]$ is upwards closed. This is clear if $U$ is empty. Let us assume $U \neq \emptyset$, and let us consider some $\left(x, x^{\prime}\right) \in U$ and some $y \in X$ with $\sup ^{(2)}\left(x, x^{\prime}\right) \leq y$. Then $x \leq y$ and $x^{\prime} \leq y$, hence, $\left(x, x^{\prime}\right) \leq_{X \times X}(y, y)$. As $U$ is upwards closed we conclude $(y, y) \in U$, hence, $y=\sup ^{(2)}(y, y) \in \sup ^{(2)}[U]$. We have shown that $\sup ^{(2)}[U]$ is upwards closed. Now let us assume that $S \subseteq X$ is a directed set such that $\sup (S)$ exists and is an element of $\sup ^{(2)}[U]$. Then the set $P:=\{(s, s) \in X \times X: s \in S\}$ is a $\leq X \times X$-directed set whose $\leq X \times X$-supremum exists and is equal to $(\sup (S), \sup (S))$. Remember that $\sup (S) \in$ $\sup ^{(2)}[U]$. Let $\left(x, x^{\prime}\right) \in U$ be an element of $U$ with $\sup (S)=\sup ^{(2)}\left(x, x^{\prime}\right)$. We conclude that $x \leq \sup (S)$ and $x^{\prime} \leq \sup (S)$, hence, $\left(x, x^{\prime}\right) \leq_{X \times X}(\sup (S), \sup (S))$. As $U$ is upwards closed we conclude $(\sup (S), \sup (S)) \in U$. Thus, $\sup _{\leq X \times X}(P) \in U$. As $U$ is Scott open we obtain $P \cap U \neq \emptyset$. Thus, there exists some $y \in S$ with $(y, y) \in U$. This implies $y=\sup ^{(2)}(y, y) \in \sup ^{(2)}[U]$. Thus, $S \cap \sup ^{(2)}[U] \neq \emptyset$. We have shown that $\sup ^{(2)}[U]$ is Scott open.

Corollary 5.4 Let $(X, \leq)$ be a sup semilattice. Then the function $\sup ^{(2)}: X \times X \rightarrow X$ $i$ open with respect to the product topology on $X \times X$ of the Scott topologies on both copies of $X$ (on the left hand side) and the Scott topology on $X$ (on the right hand side).

Proof This follows from Proposition 5.3 and from Lemma 4.2.

\section{Sobriety and the binary supremum function}

In this section we present the observation by Wei and Shouli [14] that a slightly weaker property than continuity of the binary supremum function with respect to the product topology is equivalent to the property "sober", for suitable posets. The following definitions are copied from [6, Sect. O-5], [7, Sect. 8.2]. Let $X$ be a topological space. We call a subset $C \subseteq X$ irreducible if it is nonempty and, for any closed subsets $A, B \subseteq X$ the following implication is true: if $C \subseteq A \cup B$ then $C \subseteq A$ or $C \subseteq B$. It is clear that the closure $\operatorname{cl}\left(\left\{x_{0}\right\}\right)$ of a point $x_{0}$ is irreducible, for any $x_{0} \in X$. A topological space $X$ (or its topology) is called sober if it is a $T_{0}$ space and for every irreducible closed subset $C \subseteq X$ there exists a point $x_{0} \in X$ with $C=\operatorname{cl}\left(\left\{x_{0}\right\}\right)$. Note that the $T_{0}$ property of a topological space $X$ implies for $x_{0}, x_{1} \in X$ : if $\operatorname{cl}\left(\left\{x_{0}\right\}\right)=\operatorname{cl}\left(\left\{x_{1}\right\}\right)$ then $x_{0}=x_{1}$.

We wish to analyze which posets that are dcpos and sup semilattices (compare Lemma 3.1) have a sober Scott topology. The following lemma is implicitly contained in the proof of [6, Proposition II-1.11]. 
Lemma 6.1 Let $(X, \leq)$ be a dcpo and consider on $X$ the Scott topology. For a closed subset $C \subseteq X$ the following two conditions are equivalent.

\section{1. $C$ is directed.}

2. There exists a point $x_{0} \in X$ with $C=\operatorname{cl}\left(\left\{x_{0}\right\}\right)$.

Proof Let $x_{0} \in X$. On the one hand, in Example 3.4 we already mentioned that $\operatorname{cl}\left(\left\{x_{0}\right\}\right)=\downarrow\left\{x_{0}\right\}$. And it is clear that the set $\downarrow\left\{x_{0}\right\}$ is directed. On the other hand, if a set $C$ is directed then $\sup (C)$ exists because we assume that $(X, \leq)$ is a dcpo. And if $C$ is closed and directed then $\sup (C) \in C$. But in that case it is clear that $C=\downarrow\{\sup (C)\}$.

It is well known that, if $(X, \leq)$ is a dcpo and a sup semilattice and the function $\sup ^{(2)}: X \times X \rightarrow X$ is continuous with respect to the product topology on $X$, then $X$ with the Scott topology is sober; see [5, Corollary II.1-12], [6, Corollary II1.12]. Wei and Shouli [14] observed that by weakening the continuity requirement on $\sup ^{(2)}$ one can obtain a condition that is equivalent to sobriety, under sufficiently strong further assumptions. The following theorem was essentially stated by them with slightly different assumptions.

Theorem 6.2 Let $(X, \leq)$ be a poset that is a dcpo and a sup semilattice. Then the following two conditions are equivalent.

\section{The Scott topology $\sigma(X, \leq)$ is sober.}

2. For every Scott open subset $W \subsetneq X$ whose complement is irreducible, the set $\left(\sup ^{(2)}\right)^{-1}[W] \subseteq X \times X$ is open in the product topology on $X \times X$.

Proof "(1) $\Rightarrow(2)$ ": Let $(X, \sigma(X, \leq))$ be sober. Let $W \subsetneq X$ be a proper Scott open subset of $X$ whose complement $C:=X \backslash W$ is irreducible. Let $x, y \in X$ be two elements with $\sup ^{(2)}(x, y)=: z \in W$. We wish to show that there are Scott open subsets $U, V \subseteq X$ with $x \in U, y \in V$ and $\sup ^{(2)}[U \times V] \subseteq W$. As $C$ is irreducible and $X$ is sober there exists a point $c \in X$ with $C=\operatorname{cl}(\{c\})$. If $x$ and $y$ would satisfy $x \leq c$ and $y \leq c$ then their supremum $z$ would satisfy $z \leq c$ as well, that is, $z \in \downarrow\{c\}=$ $\operatorname{cl}(\{c\})=C$. Thus, we observe that $x \not \leq c$ or $y \not \leq c$. Without loss of generality we assume $x \not c$, hence, $x \notin \downarrow\{c\}=\operatorname{cl}(\{c\})=C$, hence, $x \in W$. But now the open sets $U:=W$ and $V:=X$ satisfy $x \in U, y \in V$ and $\sup ^{(2)}[U \times V]=\sup ^{(2)}[W \times X] \subseteq W$.

"(2) $\Rightarrow(1)$ ": Let us assume that (2) is true. Let $C \subseteq X$ be a closed and irreducible subset of $X$. According to Lemma 6.1 it is sufficient to show that $C$ is directed. Let us consider two elements $x, y \in C$. We claim that the supremum $z:=\sup ^{(2)}(x, y)$ is an element of $C$. Suppose that this is not the case. Then $z \in W:=X \backslash C$. By Condition (2) there exist Scott open subsets $U, V \subseteq X$ with $x \in U, y \in V$ and $\sup ^{(2)}[U \times V] \subseteq W$. As $U$ and $V$ are upwards closed we obtain $\sup ^{(2)}[U \times V]=U \cap V$. Hence, we have $U \cap V \subseteq W$. For the closed sets $A:=X \backslash U$ and $B:=X \backslash V$ that means $C \subseteq A \cup B$. As $C$ is irrreducible this implies $C \subseteq A$ or $C \subseteq B$, hence, $U \subseteq W$ or $V \subseteq W$. We conclude $x \in W$ or $y \in W$, in contradiction to $x, y \in C$. 


\section{Continuity or discontinuity of the supremum function with respect to the product topology on a complete lattice}

Let $(X, \leq)$ be a sup semilattice. Proposition 5.3 says that the binary supremum function is continuous with respect to the Scott topology on $X \times X$. This leads to the question whether it is necessarily even continuous with respect to the product topology on $X \times X$ as well. This question was answered negatively by Isbell.

Theorem 7.1 (Isbell $[10,11])$ There exists a complete lattice $(Z, \leq)$ such that the binary supremum function $\sup ^{(2)}: Z \times Z \rightarrow Z$ is not continuous with respect to the product topology on $Z \times Z$ of the Scott topology on each copy of $Z$ (on the left hand side) and the Scott topology on $Z$ (on the right hand side).

In fact, in [10] Isbell constructed a complete lattice such that this lattice with the Scott topology is not sober. It follows from [5, Corollary II.1-12] as well as [6, Corollary II-1.12] or Theorem 6.2 that the binary supremum function on this lattice is not continuous with respect to the product topology. This result by Isbell leads to the question (posed by Hoffmann, according to [11]) whether there exists a sober complete lattice such that the binary supremum function is discontinuous with respect to the product topology. This question was answered positively by Isbell, who constructed such a lattice [11].

The observation described in the following lemma was made by Isbell in [11] for complete lattices. This fact was rediscovered by the author [8, Proof of Theorem 4.2], who at the time of writing [8] was not aware of the articles [10,11] by Isbell.

Lemma 7.2 Let $\left(X, \leq_{X}\right)$ and $\left(Y, \leq_{Y}\right)$ be sup semilattices such that $\perp_{X}:=\inf (X)$ and $\perp_{Y}:=\inf (Y)$ exist and such that the product topology on $X \times Y$ is different from the Scott topology on $X \times Y$. Then $Z:=X \times Y$ with $\leq_{X \times Y}$ is a sup semilattice such that the binary supremum function $\sup ^{(2)}: Z \times Z \rightarrow \bar{Z}$ is discontinuous with respect to the product topology on $Z$.

For completeness sake we give the proof. First, we formulate and prove another simple lemma.

Lemma 7.3 Let $\left(X, \leq_{X}\right)$ and $\left(Y, \leq_{Y}\right)$ be posets. For any $x \in X$ the function $g_{x}$ : $Y \rightarrow X \times Y$ defined by $g_{x}(y):=(x, y)$ is Scott continuous, and for any $y \in Y$ the function $g_{y}: X \rightarrow X \times Y$ defined by $g_{y}(x):=(x, y)$ is Scott continuous.

Proof This follows directly by applying Lemma 5.2.

Proof of Lemma 7.2 By Lemma 4.1 it is clear the $\left(Z, \leq_{X \times Y}\right)$ is a sup semilattice. Let $E \subseteq Z=X \times Y$ be a subset that is open in the Scott topology on $X \times Y$ but not open in the product topology on $X \times Y$. We are going to show that the preimage

$$
D:=\left(\sup ^{(2)}\right)^{-1}[E]=\left\{\left(z, z^{\prime}\right) \in Z \times Z: \sup ^{(2)}\left(z, z^{\prime}\right) \in E\right\}
$$

of $E$ under the function sup ${ }^{(2)}$ is not open in the product topology on $Z \times Z$ (of the Scott topologies on each copy of $Z$ ). For the sake of a contradiction, let us assume that 
$D$ is open in the product topology on $Z \times Z$. We are going to show that this assumption would imply that $E$ is open in the product topology on $Z=X \times Y$.

Let us fix some element $\left(x^{(0)}, y^{(0)}\right) \in E$. It is sufficient to show that the assumption that $D$ is open in the product topology on $Z \times Z$ implies that there exist a Scott open subset $F \subseteq X$ and a Scott open subset $G \subseteq Y$ with $x^{(0)} \in F$, with $y^{(0)} \in G$ and with $F \times G \subseteq E$. How do we arrive at such sets? Note that $\sup ^{(2)}\left(\left(x^{(0)}, \perp_{Y}\right),\left(\perp_{X}, y^{(0)}\right)\right)=$ $\left(x^{(0)}, y^{(0)}\right) \in E$, hence,

$$
\left(\left(x^{(0)}, \perp_{Y}\right),\left(\perp_{X}, y^{(0)}\right)\right) \in D .
$$

Our assumption that $D$ is open in the product topology on $Z \times Z$ implies that there exist two Scott open subsets $D_{1}, D_{2} \subseteq Z$ with $\left(x^{(0)}, \perp_{Y}\right) \in D_{1}$, with $\left(\perp_{X}, y^{(0)}\right) \in D_{2}$, and with $D_{1} \times D_{2} \subseteq D$. By Lemma 7.3 the sets

$$
\begin{aligned}
& F:=\left\{x \in X:\left(x, \perp_{Y}\right) \in D_{1}\right\}, \\
& G:=\left\{y \in Y:\left(\perp_{X}, y\right) \in D_{2}\right\} .
\end{aligned}
$$

are Scott open subsets of $X$ resp. of $Y$. It is clear that $x^{(0)} \in F$ and $y^{(0)} \in G$. Finally, we claim $F \times G \subseteq E$. Indeed, for $x \in F$ and $y \in G$ we obtain $\left(x, \perp_{Y}\right) \in D_{1}$ and $\left(\perp_{X}, y\right) \in D_{2}$, hence,

$$
(x, y)=\sup ^{(2)}\left(\left(x, \perp_{Y}\right),\left(\perp_{X}, y\right)\right) \in \sup ^{(2)}\left[D_{1} \times D_{2}\right] \subseteq E .
$$

This shows $F \times G \subseteq E$.

Proof of Theorem 7.1 The complete lattice $\left(X, \leq_{X}\right)$ defined in Example 4.7 (compare Lemma 4.9) and the complete lattice $\left(Y, \leq_{Y}\right)$ where $Y$ is the set of all Scott open subsets of $X$ and $\leq_{Y}$ is set-theoretic inclusion (see Example 3.2) satisfy the assumptions of Lemma 7.2; see Lemmas 4.5 and 4.10.

In view of Theorem 7.1 one might even suspect that the binary supremum function is necessarily discontinuous with respect to the product topology if the product topology is different from the Scott topology. The question whether this is the case or not was posed by an anonymous referee of [8]. The following theorem shows that this is not the case. The binary supremum function can be continuous with respect to the product topology even if the product topology is strictly weaker than the Scott topology.

Theorem 7.4 There exists a complete lattice $(Z, \leq)$ such that the product topology on $Z \times Z$ of the Scott topology on each copy of $Z$ is different from the Scott topology on $Z \times Z$, and the binary supremum function $\sup ^{(2)}: Z \times Z \rightarrow Z$ is still continuous with respect to the product topology on $Z \times Z$ of the Scott topology on each copy of $Z$ (on the left hand side) and the Scott topology on $Z$ (on the right hand side).

Proof Let $\left(X, \leq_{X}\right)$ and $\left(Y, \leq_{Y}\right)$ be complete lattices as in Proposition 4.6. We will construct a suitable $Z$ as the direct sum of $X$ and $Y$ except that we add an additional 


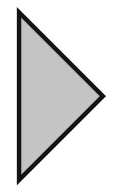

X

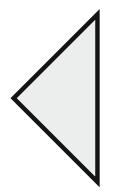

Y

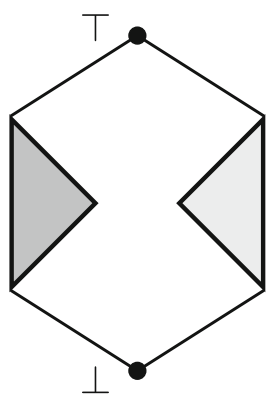

Z

Fig. 2 The complete lattice $Z$ in the proof of Theorem 7.4, constructed out of two complete lattices $X$ and $Y$

smallest and an additional largest element in order to make $Z$ a complete lattice. Formally, this can be done as follows. Let

$$
Z:=(X \times\{0\}) \cup(Y \times\{1\}) \cup\{\perp, \top\}
$$

where $\perp$ and $\top$ are different elements that are not elements of $(X \times\{0\}) \cup(Y \times\{1\})$, and let the binary relation $\leq_{Z}$ on $Z$ be defined by

$$
\begin{aligned}
& z \leq_{Z} z^{\prime}: \Longleftrightarrow z=\perp \text { or } z^{\prime}=\top \text { or } \\
& \left(\exists x, x^{\prime} \in X\right)\left(z=(x, 0) \text { and } z^{\prime}=\left(x^{\prime}, 0\right) \text { and } x \leq_{X} x^{\prime}\right) \text { or } \\
& \left(\exists y, y^{\prime} \in Y\right)\left(z=(y, 1) \text { and } z^{\prime}=\left(y^{\prime}, 1\right) \text { and } y \leq_{Y} y^{\prime}\right),
\end{aligned}
$$

for all $z, z^{\prime} \in Z$.

We come to the correctness proof. In fact, for notational simplicity, in the following we will identify $X$ with $X \times\{0\}$ and $Y$ with $Y \times\{1\}$. In other words, we will assume that $\perp \neq \top$ and that the three sets $X, Y$, and $\{\perp, \top\}$ are pairwise disjoint. Then the pair $\left(Z, \leq_{Z}\right)$ consisting of a set $Z$ and a binary relation $\leq_{Z}$ on $Z$ can be defined as follows.

$$
Z=X \cup Y \cup\{\perp, \top\}
$$

and

$$
\begin{aligned}
z \leq_{Z} z^{\prime}: \Longleftrightarrow & z=\perp \text { or } z^{\prime}=\top \text { or } \\
& \left(z \in X \text { and } z^{\prime} \in X \text { and } z \leq_{X} z^{\prime}\right) \text { or } \\
& \left(z \in Y \text { and } z^{\prime} \in Y \text { and } z \leq_{Y} z^{\prime}\right),
\end{aligned}
$$

for all $z, z^{\prime} \in Z$. See Fig. 2 . 
It is straightforward to check that $\left(Z, \leq_{Z}\right)$ is a partial order. And for any subset $S \subseteq Z$ the supremum of $S$ exists:

$$
\sup _{\leq Z}(S)= \begin{cases}\perp & \text { if } S \subseteq\{\perp\} \\ \sup _{\leq X}(S \cap X) & \text { if } S \subseteq X \cup\{\perp\} \text { and } S \cap X \neq \emptyset \\ \sup _{\leq_{Y}}(S \cap Y) & \text { if } S \subseteq Y \cup\{\perp\} \text { and } S \cap Y \neq \emptyset \\ \top & \text { if } S \nsubseteq X \cup\{\perp\} \text { and } S \nsubseteq Y \cup\{\perp\}\end{cases}
$$

Hence, $\left(Z, \leq_{Z}\right)$ is a complete lattice. Next, we show that the Scott topology on $Z \times Z$ is strictly finer than the product topology on $Z \times Z$ (that is, than the product topology on $Z \times Z$ of the Scott topology on each copy of $Z$ ). Let $E \subseteq X \times Y$ be a Scott open subset of $X \times Y$ that is not open with respect to the product topology on $X \times Y$. We claim that the set

$$
G:=E \cup((Y \cup\{\top\}) \times Z) \cup(Z \times(X \cup\{\top\}))
$$

is a Scott open subset of $Z \times Z$ and not open with respect to the product topology on $Z \times Z$.

Let us show that $G$ is $\leq_{Z \times Z}$-upwards closed. The subsets $(Y \cup\{\top\}) \times Z$ and $Z \times(X \cup\{\top\})$ are obviously $\leq Z \times Z$-upwards closed. Let us consider some $(x, y) \in$ $E$ and some $\left(z_{1}, z_{2}\right) \in Z \times Z$ with $(x, y) \leq_{Z \times Z}\left(z_{1}, z_{2}\right)$. It is sufficient to show $\left(z_{1}, z_{2}\right) \in G$. From $E \subseteq X \times Y$ we conclude $z_{1} \in X \cup\{\top\}$ and $z_{2} \in Y \cup\{\top\}$ and, furthermore, $z_{1} \in X \Rightarrow x \leq X \quad z_{1}$, and, similarly, $z_{2} \in Y \Rightarrow y \leq_{Y} z_{2}$. If $z_{1}=\top$ then $\left(z_{1}, z_{2}\right) \in(Y \cup\{\top\}) \times Z \subseteq G$, and similarly, if $z_{2}=\top$ then $\left(z_{1}, z_{2}\right) \in$ $Z \times(X \cup\{\top\}) \subseteq G$. So, let us assume that $z_{1} \in X$ and $z_{2} \in Y$. Then $x \leq X \quad z_{1}$ and $y \leq_{Y} z_{2}$, hence $(x, y) \leq_{X \times Y}\left(z_{1}, z_{2}\right)$. As $E$ is $\leq_{X \times Y}$-upwards closed we conclude $\left(z_{1}, z_{2}\right) \in E \subseteq G$. We have shown that $G$ is $\leq Z \times Z$-upwards closed.

Next, let $S \subseteq Z \times Z$ be a directed set such that $\sup _{\leq Z \times Z}(S)$ is an element of $G$ (note that $\sup _{Z \times Z}(S)$ exists because $Z \times Z$ is a complete lattice by Lemma 4.1 (4)). We wish to show that $S \cap G \neq \emptyset$. For the sake of a contradiction, let us assume that $S \cap G=\emptyset$. Then $S \cap((Y \cup\{\top\}) \times Z)=\emptyset$ and $S \cap(Z \times(X \cup\{\top\}))=\emptyset$, hence, $S \subseteq(X \cup\{\perp\}) \times(Y \cup\{\perp\})$. This implies

$$
\sup _{\leq Z \times Z}(S)=\left(\sup _{\leq Z}\left(\pi_{1}[S]\right), \sup _{\leq Z}\left(\pi_{2}[S]\right)\right) \in(X \cup\{\perp\}) \times(Y \cup\{\perp\})
$$

compare Lemma 4.1 (3). With $\sup _{\leq Z \times Z}(S) \in G$ and by the definition of $G$ we obtain $\sup _{\leq Z \times Z}(S) \in E$. This, in turn, implies $\pi_{1}[S] \cap X \neq \emptyset$ (otherwise we would have $\pi_{1}[S]=\{\perp\}$, hence, also $\sup _{\leq Z}\left(\pi_{1}[S]\right)=\perp$ in contradiction to $\sup _{\leq Z \times Z}(S) \in E$ and $E \cap(\{\perp\} \times Z)=\emptyset)$ and $\pi_{2}[S] \cap Y \neq \emptyset$. As $S$ is a directed set we can conclude that $S \cap(X \times Y) \neq \varnothing$. Furthermore, the set $S \cap(X \times Y)$ is a $\leq X \times Y$-directed subset of $X \times Y$ with

$$
\sup _{\leq X \times Y}(S \cap(X \times Y))=\sup _{\leq Z \times Z}(S \cap(X \times Y))=\sup _{\leq Z \times Z}(S) \in E .
$$


As $E$ is a Scott open subset of $X \times Y$ we conclude that $S \cap(X \times Y) \cap E \neq \emptyset$, hence, $S \cap E \neq \emptyset$, hence, $S \cap G \neq \emptyset$. Contradiction. We have shown that $G$ is a Scott open subset of $Z \times Z$.

We still have to show that $G$ is not open with respect to the product topology on $Z \times Z$. For the sake of a contradiction, let us assume that $G$ is open with respect to the product topology on $Z \times Z$. We are going to show that this implies that the subset $E$ of $X \times Y$ is open with respect to the product topology on $X \times Y$. Let us consider an arbitrary element $(x, y)$ of $E$. We wish to show that there exist Scott open subsets $U^{\prime} \subseteq X$ and $V^{\prime} \subseteq Y$ with $x \in U^{\prime}, y \in V^{\prime}$, and $U^{\prime} \times V^{\prime} \subseteq E$. We make use of the fact that $(x, y) \in E$ implies $(x, y) \in G$. As by assumption $G$ is open with respect to the product topology on $Z \times Z$ there exist Scott open subsets $U \subseteq Z$ and $V \subseteq Z$ with $(x, y) \in U \times V$ and $U \times V \subseteq G$. Then the set $U^{\prime}:=U \cap X$ is a Scott open subset of $X$, and the set $V^{\prime}:=V \cap Y$ is a Scott open subset of $Y$. We observe $x \in U^{\prime}, y \in V^{\prime}$, and $U^{\prime} \times V^{\prime} \subseteq G \cap(X \times Y)=E$. Hence, $G$ is indeed not open with respect to the product topology on $Z \times Z$.

Finally, we have to show that the function $\sup ^{(2)}: Z \times Z \rightarrow Z$ is continuous with respect to the product topology on $Z \times Z$. Let $W \subseteq Z$ be a Scott open subset. It is sufficient to show that $\left(\sup ^{(2)}\right)^{-1}[W]$ is open in the product topology on $Z \times Z$. We can assume that $W$ is not empty. Let $\left(z_{1}, z_{2}\right) \in Z \times Z$ be a pair with $\sup ^{(2)}\left(z_{1}, z_{2}\right) \in W$. It is sufficient to show that there exist Scott open subsets $W_{1}, W_{2} \subseteq Z$ with $z_{1} \in W_{1}$, $z_{2} \in W_{2}$ and $W_{1} \times W_{2} \subseteq\left(\sup ^{(2)}\right)^{-1}[W]$. We distinguish several cases for $\left(z_{1}, z_{2}\right)$.

Case $1 z_{1}=\perp$. The sets $Z$ and $W$ are Scott open subsets of $Z, z_{1}=\perp \in Z$, $z_{2}=\sup ^{(2)}\left(z_{1}, z_{2}\right) \in W$, and $Z \times W \subseteq\left(\sup ^{(2)}\right)^{-1}[W]$.

Case $2 z_{2}=\perp$. This case is symmetric to Case 1 and is treated in the same way.

Case $3 z_{1}=\top$. Then $\top=\sup ^{(2)}\left(z_{1}, z_{2}\right) \in W$. The set $\{\top\}$ is a Scott open subset of $Z$ (indeed, if $S \subseteq Z$ is a subset with $\sup (S)=\top$ then $S \cap(X \cup\{\top\}) \neq \varnothing$ and $S \cap(Y \cup\{\top\}) \neq \emptyset$, and if $S$ is directed this implies $\top \in S)$. And the set $Z$ is of course a Scott open subset of $Z$ as well. Finally, we note that $z_{1} \in\{\top\}, z_{2} \in Z$, and $\{\top\} \times Z \subseteq\left(\sup ^{(2)}\right)^{-1}[W]$.

Case $4 z_{2}=\top$. This case is symmetric to Case 3 and is treated in the same way.

Case $5 z_{1} \in X$ and $z_{2} \in Y$. Then $\top=\sup ^{(2)}\left(z_{1}, z_{2}\right) \in W$. The sets $X \cup\{\top\}$ and $Y \cup\{\top\}$ are Scott open subsets of $Z, z_{1} \in X \cup\{\top\}, z_{2} \in Y \cup\{\top\}$, and $(X \cup\{\top\}) \times(Y \cup\{\top\}) \subseteq\left(\sup ^{(2)}\right)^{-1}[\{\top\}] \subseteq\left(\sup ^{(2)}\right)^{-1}[W]$.

Case $6 z_{1} \in Y$ and $z_{2} \in X$. This case is symmetric to Case 5 and is treated in the same way.

Case $7 z_{1} \in X$ and $z_{2} \in X$. By Proposition 5.3 the function $\sup ^{(2)}: Z \times Z \rightarrow Z$ is continuous with respect to the Scott topology on $Z \times Z$. Hence, $\left(\sup ^{(2)}\right)^{-1}[W]$ is a Scott open subset of $Z \times Z$. We claim that the set $W^{\prime}:=\left(\sup ^{(2)}\right)^{-1}[W] \cap(X \times X)$ is a Scott open subset of $X \times X$ (that means, Scott open with respect to $\leq X \times X$ ). This follows directly from the fact that the restriction of $\leq Z \times Z$ to $X \times X$ is identical with $\leq X \times X$. By the second condition in Proposition 4.6 the Scott topology on $X \times \bar{X}$ is identical with the product topology. Hence, the subset $W^{\prime}$ of $X \times X$ 
is open in the product topology. Furthermore $\left(z_{1}, z_{2}\right) \in W^{\prime}$. Hence, there exist $\leq X$-Scott open subsets $U_{1}, U_{2} \subseteq X$ with $z_{1} \in U_{1}, z_{2} \in U_{2}$ and $U_{1} \times U_{2} \subseteq W^{\prime}$. The sets $W_{1}:=U_{1} \cup\{\top\}$ and $W_{2}:=U_{2} \cup\{T\}$ are $\leq_{Z}$-Scott open subsets of $Z$ and satisfy $z_{1} \in W_{1}, z_{2} \in W_{2}$, and $W_{1} \times W_{2} \subseteq\left(\sup ^{(2)}\right)^{-1}[W]$.

Case $8 z_{1} \in Y$ and $z_{2} \in Y$. This case is treated in the same way as Case 7, using the third condition in Proposition 4.6 instead of the second condition.

\section{Failure of bounded completeness for the directed complete partial order of Scott continuous functions}

Let us consider some poset $(Z, \leq)$ and an arbitrary topological space $X$. We call a function $f: X \rightarrow Z$ Scott continuous if it is continuous with respect to the given topology on $X$ and the Scott topology on $Z$. Let $\mathrm{C}(X, Z)$ denote the set of all Scott continuous functions $f: X \rightarrow Z$. It is clear that the binary relation $\leq_{\mathrm{C}}$ defined on $X$ by

$$
f \leq_{\mathrm{C}} g: \Longleftrightarrow(\forall x \in X) f(x) \leq g(x)
$$

for $f, g \in \mathrm{C}(X, Z)$, is a partial order, that is, $\left(\mathrm{C}(X, Z), \leq_{\mathrm{C}}\right)$ is a poset.

Proposition 8.1 ( [13, Lemma 1-4.6], [9, Prop. 6]) Let $(Z, \leq)$ be a dcpo, and let X be an arbitrary topological space. Then $\mathrm{C}(X, Z)$ with $\leq_{\mathrm{C}}$ is a dcpo. Furthermore, if $F \subseteq \mathrm{C}(X, Z)$ is a $\leq_{\mathrm{C}}$-directed set then the function $g: X \rightarrow Z$ defined by

$$
g(x):=\sup (\{f(x): f \in F\})
$$

(this is well defined because, for any $x \in X$, the set $\{f(x): f \in F\}$ is directed and because $(Z, \leq)$ is a dcpo) is Scott continuous and the least upper bound of $F$.

Let $(Z, \leq)$ be a poset. A subset $S \subseteq Z$ is called bounded if there exists an upper bound $z \in Z$ for $S$. The poset $(Z, \leq)$ itself is called bounded if $Z$ is a bounded set. The poset $(Z, \leq)$ is called bounded complete if for any bounded subset $S \subseteq Z$ there exists a supremum of $S$ in $Z$. An element of $Z$ is called a least element of $Z$ if it is a lower bound of $Z$. Obviously, if a least element exists then it is unique. Note that any bounded complete poset $(Z, \leq)$ has a least element (the element $\sup (\emptyset)$ does the job). And note that any complete lattice is a dcpo and that a poset is a complete lattice if, and only if, it is bounded and bounded complete.

Lemma 8.2 Let $X$ be an arbitrary topological space.

1. If $(Z, \leq)$ is a bounded poset then $\left(\mathrm{C}(X, Z), \leq_{\mathrm{C}}\right)$ is a bounded poset as well.

2. If $(Z, \leq)$ is a poset with least element then $\left(\mathrm{C}(X, Z), \leq_{\mathrm{C}}\right)$ is a poset with least element as well.

Proof Let $(Z, \leq)$ be a poset. We have already seen that then $\left(\mathrm{C}(X, Z), \leq_{\mathrm{C}}\right)$ is a poset as well. 
1. Let $\top \in Z$ be an upper bound for $Z$. The constant function $f: X \rightarrow Z$ with $f(x):=\top$ for all $x \in X$ is Scott continuous, thus, an element of $\mathrm{C}(X, Z)$, and it is an upper bound for $\mathrm{C}(X, Z)$.

2. Let $\perp \in Z$ be a least element of $Z$. The constant function $f: X \rightarrow Z$ with $f(x):=\perp$ for all $x \in X$ is Scott continuous, thus, an element of $\mathrm{C}(X, Z)$, and it is a lower bound for $\mathrm{C}(X, Z)$.

The following question arises: If $(Z, \leq)$ is bounded complete, is then $\mathrm{C}(X, Z)$ bounded complete as well? This is true if the topology considered on $X$ is the Scott topology induced by a partial order relation on $X$.

Proposition 8.3 Let $\left(X, \leq_{X}\right)$ be a poset and consider on $X$ the Scott topology. If $\left(Z, \leq_{Z}\right)$ is a bounded complete poset then $\left(\mathrm{C}(X, Z), \leq_{\mathrm{C}}\right)$ is a bounded complete poset as well.

Proof Let $F \subseteq \mathrm{C}(X, Z)$ be a $\leq_{\mathrm{C}}$-bounded set. Then for each $x \in X$ the set

$$
F(x):=\{f(x): f \in F\}=\{z \in Z:(\exists f \in F) z=f(x)\}
$$

is bounded (indeed, if $h \in \mathrm{C}(X, Z)$ is a $\leq_{\mathrm{C}}$-upper bound of $F$ then, for every $x \in X$, the element $h(x) \in Z$ is a $\leq Z$-upper bound of the set $F(x))$. Since we assume $\left(Z, \leq_{Z}\right)$ to be bounded complete, we can define a function $g: X \rightarrow Z$ by

$$
g(x):=\sup (F(x))
$$

First, we show that this function $g$ is Scott continuous. We use the characterization in Lemma 5.2. First we show that $g$ is monotone. Let us consider $x, y \in X$ with $x \leq_{X} y$. As each function $f \in F$ is monotone, we have for each function $f \in F$

$$
f(x) \leq_{Z} f(y) \leq_{Z} g(y),
$$

hence, $g(x) \leq_{Z} g(y)$. Thus, $g$ is monotone. Now, let $S \subseteq X$ be a directed set such that $\sup (S)$ exists. For every $s \in S$ we have $g(s) \leq_{Z} g(\sup (S))$ because $g$ is monotone, hence, $g(\sup (S))$ is an upper bound of $g[S]$. Let $z_{0} \in Z$ be an arbitrary upper bound of $g[S]$. We wish to show $g(\sup (S)) \leq_{Z} z_{0}$. For every $f \in F$ and every $s \in S$,

$$
f(s) \leq_{Z} g(s) \leq_{Z} z_{0} .
$$

As every $f \in F$ is Scott continuous, for every $f \in F$, $\sup (f[S])$ exists and we obtain

$$
f(\sup (S))=\sup (f[S]) \leq_{Z} z_{0} .
$$

We conclude

$$
g(\sup (S))=\sup (F(\sup (S)))=\sup \{f(\sup (S)): f \in F\} \leq_{Z} z_{0} .
$$

This completes the proof of our claim that $g$ is Scott continuous. 
It is obvious that $g$ is an upper bound of $F$. If $h \in \mathrm{C}(X, Z)$ is any upper bound of $F$ then for all $x \in X$ and all $f \in F, f(x) \leq_{Z} h(x)$, hence, $g(x)=\sup \{f(x): f \in$ $F\} \leq_{Z} h(x)$, hence, $g \leq_{\mathrm{C}} h$. We have shown that $g$ is the least upper bound of $F$.

One may now speculate whether for an arbitrary topological space $X$ and an arbitrary bounded complete dcpo $Z$ (with least element) the dcpo $\mathrm{C}(X, Z)$ is bounded complete as well. For example, Edalat [4, p. 502] claims that this is the case by writing: "For any topological space $Z$ and any bounded complete dcpo $D$ with bottom $\perp$, let $Z \rightarrow D$ be the bounded complete dcpo of Scott continuous functions from $Z$ to $D$." But we are going to show that in general this is not the case; see Theorem 8.5 below.

Remark 8.4 The claim by Edalat, cited above, is a crucial step in his argument in [4] why the notion of the L-derivative, introduced by him in [4], is well-defined. As this claim is incorrect the reader might now be worried whether the $L$-derivative is welldefined at all. But indeed it is. One can replace the cited claim by a different argument; compare [9].

Theorem 8.5 There exist a topological space $X$ and a complete lattice $(Z, \leq)$ with the following properties:

1. $\mathrm{C}(X, Z)$ is a bounded dcpo with least element, but not bounded complete.

2. There exist two functions $f, g \in \mathrm{C}(X, Z)$ with the following two properties.

(a) The set $\{f, g\}$ does not have $a \leq_{\mathrm{C}}$-supremum in $\mathrm{C}(X, Z)$.

(b) The pointwise supremum of $f$ and $g$, that is, the function $h: X \rightarrow Z$ defined by $h(x):=\sup \{f(x), g(x)\}$, is not Scott continuous.

It is clear that Property (a) in Theorem 8.5 implies Property (b). The converse in not so obvious, but true as well, as the following lemma shows. It will be used in the proof of Theorem 8.5.

Lemma 8.6 For any topological space $X$, any bounded sup semilattice $(Z, \leq Z)$, and any two functions $f, g \in \mathrm{C}(X, Z)$ the following two conditions are equivalent.

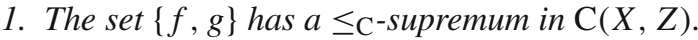

2. The pointwise supremum $h: X \rightarrow Z$ of $f$ and $g$ defined by

$$
h(x):=\sup \{f(x), g(x)\}
$$

for all $x \in X$, is Scott continuous.

Furthermore, if one (and then both) of these two conditions is satisfied then the pointwise supremum of $f$ and $g$ is the $\leq_{\mathrm{C}}$-supremum of $\{f, g\}$ in $\mathrm{C}(X, Z)$.

Proof Fix some functions $f, g \in \mathrm{C}(X, Z)$, and let $h: X \rightarrow Z$ be the pointwise supremum of $f$ and $g$.

"2 $\Rightarrow 1$ ": If $h$ is Scott continuous and $r \in \mathrm{C}(X, Z)$ any function with $f \leq_{\mathrm{C}} r$ and $g \leq_{\mathrm{C}} r$ then $h \leq_{\mathrm{C}} r$. Thus, in this case $h$ is a supremum of $\{f, g\}$ in $\mathrm{C}(X, Z)$. 
"1 $\Rightarrow 2$ ": For the converse direction, let us first show the following claim.

Claim. For any $x_{0} \in X$ there exists a function $s_{x_{0}} \in \mathrm{C}(X, Z)$ with $f \leq_{\mathrm{C}} s_{x_{0}}$, with $g \leq_{\mathrm{C}} s_{x_{0}}$, and with $s_{x_{0}}\left(x_{0}\right)=\sup \left\{f\left(x_{0}\right), g\left(x_{0}\right)\right\}$.

Proof of this claim. Let $\top:=\sup (Z)$ (this exists because $(Z, \leq$ ) is assumed to be bounded). Let us fix an arbitrary point $x_{0} \in X$. Let us define a "step function" $s_{x_{0}}$ : $X \rightarrow Z$ by

$$
s_{x_{0}}(x):= \begin{cases}\sup \left\{f\left(x_{0}\right), g\left(x_{0}\right)\right\} & \text { if } x \in \operatorname{cl}\left(\left\{x_{0}\right\}\right) \\ \top & \text { otherwise }\end{cases}
$$

We claim that this function has the desired properties. First, we claim that $s_{x_{0}}$ is Scott continuous. This is clear if $\sup \left\{f\left(x_{0}\right), g\left(x_{0}\right)\right\}=\top$ because in that case $s_{x_{0}}$ is the constant function with value $\top$. Also in the case $\sup \left\{f\left(x_{0}\right), g\left(x_{0}\right)\right\} \neq \top$ for any Scott open subset $V \subseteq Z$ the set

$$
s_{x_{0}}^{-1}[V]= \begin{cases}\emptyset & \text { if } V=\varnothing, \\ X \backslash \operatorname{cl}\left(\left\{x_{0}\right\}\right) & \text { if } V \neq \varnothing \text { and } \sup \left\{f\left(x_{0}\right), g\left(x_{0}\right)\right\} \notin V, \\ X & \text { if } \sup \left\{f\left(x_{0}\right), g\left(x_{0}\right)\right\} \in V\end{cases}
$$

is an open subset of $X$. Hence, the function $s_{x_{0}}$ is Scott continuous, thus, an element of $\mathrm{C}(X, Z)$. We claim that furthermore $f \leq_{\mathrm{C}} s_{x_{0}}$. Indeed, if $x \notin \operatorname{cl}\left(\left\{x_{0}\right\}\right)$ then $f(x) \leq_{Z}$ $\top=s_{x_{0}}(x)$. Let us now consider the case $x \in \operatorname{cl}\left(\left\{x_{0}\right\}\right)$. The set

$$
V:=\left\{z \in Z: z \not \leq Z f\left(x_{0}\right)\right\}
$$

is a Scott open subset of $Z$ by Example 3.4. As $f: X \rightarrow Z$ is Scott continuous, the set $U:=f^{-1}[V]$ is an open subset of $X$. It is clear that $x_{0} \notin U$. Hence, $x \in \operatorname{cl}\left(\left\{x_{0}\right\}\right) \subseteq$ $X \backslash U$, that is, $x \notin U$. This means $f(x) \leq_{Z} f\left(x_{0}\right)$. We obtain

$$
f(x) \leq_{Z} f\left(x_{0}\right) \leq_{Z} \sup \left\{f\left(x_{0}\right), g\left(x_{0}\right)\right\}=s_{x_{0}}(x) .
$$

We have shown $f \leq_{\mathrm{C}} s_{x_{0}}$. By the same argument $g \leq_{\mathrm{C}} s_{x_{0}}$ follows. Finally, it is clear that $s_{x_{0}}\left(x_{0}\right)=\sup \left\{f\left(x_{0}\right), f\left(x_{0}\right)\right\}$. We have shown the claim.

Now let us assume that the set $\{f, g\}$ has a supremum in $\mathrm{C}(X, Z)$. Let us call this supremum $t$. Let us consider some $x_{0} \in X$. Clearly $h\left(x_{0}\right)=\sup \left\{f\left(x_{0}\right), g\left(x_{0}\right)\right\} \leq_{Z}$ $t\left(x_{0}\right)$. According to the claim that we have just proved, there exists a function $s_{x_{0}} \in \mathrm{C}(X, Z)$ with $f \leq_{\mathrm{C}} s_{x_{0}}$, with $g \leq_{\mathrm{C}} s_{x_{0}}$ and with $s_{x_{0}}\left(x_{0}\right)=\sup \left\{f\left(x_{0}\right), g\left(x_{0}\right)\right\}$. This implies $t=\sup \{f, g\} \leq_{\mathrm{C}} \quad s_{x_{0}}$ and in particular $t\left(x_{0}\right) \leq_{Z} \quad s_{x_{0}}\left(x_{0}\right)=$ $\sup \left\{f\left(x_{0}\right), g\left(x_{0}\right)\right\}=h\left(x_{0}\right)$. Thus, we do not only have $h\left(x_{0}\right) \leq_{Z} t\left(x_{0}\right)$ but also $t\left(x_{0}\right) \leq_{Z} h\left(x_{0}\right)$, thus, $t\left(x_{0}\right)=h\left(x_{0}\right)$. As this is true for all $x_{0} \in X$, we have $t=h$, that is, $t$ must be the pointwise supremum of $f$ and $g$.

Proof of Theorem 8.5 Let $(Z, \leq)$ be a complete lattice as in Theorem 7.1. Then $Z$ is bounded $(\sup (Z)$ is an upper bound) as well as bounded complete and directed 
complete (every subset has a supremum), and it has a least element. For $X$ we choose $Z \times Z$ with the product topology of the Scott topologies on each copy of $Z$. By Proposition $8.1 \mathrm{C}(X, Z)$ is a dcpo and by Lemma 8.2 it is bounded and has a least element. Once we have shown that there exist two functions $f, g \in \mathrm{C}(X, Z)$ that do not have a supremum in $\mathrm{C}(X, Z)$ we have shown that $\mathrm{C}(X, Z)$ is not bounded complete.

In fact, for $f$ and $g$ we can take the projection functions $\pi_{1}, \pi_{2}: X=Z \times Z \rightarrow Z$ defined by $\pi_{i}\left(z_{1}, z_{2}\right):=z_{i}$ for $i=1,2$ and all $z_{1}, z_{2} \in Z$. For $i=1,2$ the projection function $\pi_{i}$ is continuous with respect to the product topology on $X=Z \times Z$, thus, $\pi_{i} \in \mathrm{C}(X, Z)$. Note that the function $\sup ^{(2)}$ is the pointwise supremum of $\pi_{1}$ and $\pi_{2}$. As we have chosen $Z$ to be a complete lattice as in Theorem 7.1 and $X$ to be $Z \times Z$ with the product topology, the function $\sup ^{(2)}$ is not an element of $\mathrm{C}(X, Z)$, that is, $\sup ^{(2)}$ is not continuous. Thus, the pointwise supremum of the continuous functions $\pi_{1}: X \rightarrow Z$ and $\pi_{2}: X \rightarrow Z$ is not continuous. And, according to Lemma 8.6, the set $\left\{\pi_{1}, \pi_{2}\right\}$ does not have a $\leq_{\mathrm{C}}$-supremum in $\mathrm{C}(X, Z)$. This ends the proof of Theorem 8.5.

Acknowledgements The author was supported by the EU grant FP7-PEOPLE-2011-IRSES No. 294962: COMPUTAL. I thank Xiaoquan Xu for telling me about the Isbell lattice [10] and an anonymous referee of an earlier version of the paper for useful comments.

Funding Open Access funding enabled and organized by Projekt DEAL.

Open Access This article is licensed under a Creative Commons Attribution 4.0 International License, which permits use, sharing, adaptation, distribution and reproduction in any medium or format, as long as you give appropriate credit to the original author(s) and the source, provide a link to the Creative Commons licence, and indicate if changes were made. The images or other third party material in this article are included in the article's Creative Commons licence, unless indicated otherwise in a credit line to the material. If material is not included in the article's Creative Commons licence and your intended use is not permitted by statutory regulation or exceeds the permitted use, you will need to obtain permission directly from the copyright holder. To view a copy of this licence, visit http://creativecommons.org/licenses/by/4.0/.

\section{References}

1. Abramsky, S., Jung, A.: Domain theory. In: Abramsky, S., Gabbay, D.M., Maibaum, T.S.E. (eds.) Handbook of Logic in Computer Science, vol. 3, pp. 1-168. Clarendon Press, UK (1994)

2. Chen, Y., Kou, H., Lyu, Z.: Two topologies on the lattice of Scott closed subsets. Topol. Appl. 306, 107918 (2022)

3. de Brecht, M.: On countability: the compact completed sequence. Blog by Jean Goubault-Larrecq: https://projects.lsv.ens-cachan.fr/topology/?page_id=1852 (2019)

4. Edalat, A.: A continuous derivative for real-valued functions. In: Cooper, S.B., Löwe, B., Sorbi, A. (eds.) New Computational Paradigms: Changing Conceptions of What is Computable, pp. 493-519. Springer, New York (2008)

5. Gierz, G., Hofmann, K.H., Keimel, K., Lawson, J.D., Mislove, M., Scott, D.S.: A Compendium of Continuous Lattices. Springer-Verlag, Berlin, Heidelberg, New York (1980)

6. Gierz, G., Hofmann, K.H., Keimel, K., Lawson, J.D., Mislove, M., Scott, D.S.: Continuous Lattices and Domains. Cambridge University Press, Cambridge (2003)

7. Goubault-Larrecq, J.: Non-Hausdorff Topology and Domain Theory. Cambridge University Press, Cambridge (2013) 
8. Hertling, P.: Two counterexamples concerning the Scott topology on a partial order. Arxiv: $1607.04128 \mathrm{v} 1$ (2016)

9. Hertling, P.: Clarke's generalized gradient and Edalat's L-derivative. J. Log. Anal. 9, c1, 21 (2017)

10. Isbell, J.: Completion of a construction of Johnstone. Proc. Am. Math. Soc. 85(3), 333-334 (1982)

11. Isbell, J.: Discontinuity of meets and joins. In: Continuous Lattices and Their Applications (Bremen, 1982), Volume 101 of Lecture Notes in Pure and Appl. Math., pp. 151-154. Dekker, New York (1985)

12. Johnstone, P.T.: Scott is not always sober. In: Continuous Lattices, Proc. Conf., Bremen 1979, Lect. Notes Math. vol. 871, pp. 282-283 (1981)

13. Keimel, K., Lawson, J.D.: Continuous and completely distributive lattices. In: Lattice Theory: Special Topics and Applications, vol. 1, pp. 5-53. Birkhäuser/Springer (2014)

14. Wei, H., Shouli, J.: Remarks on the sobriety of Scott topology and weak topology on posets. Comment. Math. Univ. Carolin. 43(3), 531-535 (2002)

Publisher's Note Springer Nature remains neutral with regard to jurisdictional claims in published maps and institutional affiliations. 